Personal non-life insurance decisions and the welfare loss from flat deductibles

Steffensen, Mogens; Thøgersen, Julie

Published in:

ASTIN Bulletin

DOI:

$10.1017 / \mathrm{asb} .2019 .40$

Publication date:

2019

Document version

Peer reviewed version

Document license:

Other

Citation for published version (APA):

Steffensen, M., \& Thøgersen, J. (2019). Personal non-life insurance decisions and the welfare loss from flat deductibles. ASTIN Bulletin, 49(1), 85-116. https://doi.org/10.1017/asb.2019.40 


\title{
Personal non-life insurance decisions and the welfare loss from flat deductibles.
}

\author{
Mogens Steffensen ${ }^{1}$ and Julie Thøgersen ${ }^{2}$ \\ ${ }^{1}$ Department of Mathematical Sciences, Copenhagen University. \\ ${ }^{2}$ Department of Mathematics, Aarhus University.
}

\begin{abstract}
We view the retail non-life insurance decision from the perspective of the insured. We formalize different consumption-insurance problems depending on the flexibility of the insurance contract. For exponential utility and power utility we find the optimal flexible insurance decision or insurance contract. For exponential utility we also find the optimal position in standard contracts that are less flexible and therefore, for certain non-linear pricing rules, lead to a welfare loss for the individual insuree compared to the optimal flexible insurance decision. For the exponential loss distribution, we quantify a significant welfare loss. This calls for product development in the retail insurance business.
\end{abstract}

Keywords: exponential utility, HJB equation, insurance pricing, product design, compound Poisson loss process.

\section{Introduction}

The standard marketed retail insurance contract has a fixed amount deductible where the deductible is independent of the size of the loss. This simple product structure is sufficient but only for the simple pricing mechanism where the loading to the expectation is a plain factor to the expectation, also called the expectation principle. Both theoretically and practically other pricing principles are more relevant but the main focus has still been on the fixed deductible. We study how this mismatch of development between pricing and product design affects individuals in the insurance market. We therefore formulate and solve the personal consumptioninsurance problem with respect to a non-life risk modelled by a compound Poisson process with the objective to maximize utility of consumption. Two types of insurance products are considered: one with an optimal flexible, claim-dependent deductible and one with an optimal constant fixed amount deductible (the standard product). These are compared by measuring the welfare loss of an individual, which we define as the monetary compensation the individual requires in addition to the standard product in order to be indifferent between that and the optimal flexible product. For the expected value premium principle, henceforth referred to as the linear pricing principle due to its linear relation to the expected value of 
the risk, the constant deductible is known to be optimal and there is no welfare loss from being offered a standard constant deductible contract. For a certain formalization of the variance pricing principle, we find a log-power deductible to be optimal, and for a certain formalization of the Esscher pricing principle, we find a linear deductible to be optimal. In both of these cases a welfare loss arises if the individual is offered a standard constant deductible contract.

Stochastic control theory has been applied intensively to decision problems in insurance over the last decades. In life and pension insurance the applications are in two separate directions: The Asset-Liability Management decisions of a pension fund and an individual's financial consumption-investment-insurance decisions. In some formulations the two directions have much in common. In non-life insurance, most applications are to the decision making of the insurance company. Here the focus has been on the decisions concerning reinsurance, investments, premium collection, and dividends paid to the owners. A standard objective is the expected accumulated present value of future dividend payouts until ruin. There has been less focus on the non-life insurance decisions made by the individual over her lifecycle in the sense of personal financial consumption-investment-insurance decisions with respect to non-life risks. Our study is a contribution in the latter direction. To formulate an individual's non-life risk decision, we need to think carefully about how the wealth process is influenced by non-life risk, what can be controlled and how, and what is the objective function. In the next three paragraphs we address these three ingredients of the control problem one by one in order to make our standing point clear.

The compound Poisson process is well-established as a benchmark for modelling a portfolio of non-life risks. This is also called a collective risk model. We choose the same model for an individual's non-life risks. This is consistent in the sense that if an individual risk process follows a compound Poisson process, then an aggregation of those in a portfolio is also a compound Poisson process. If further the individual risk processes are homogeneous across individuals, then the collective follows a compound Poisson process with the same claim amount distribution but with a claim intensity corresponding to the individual claim intensity times the number of individuals.

The individual can typically control the non-life risk by the choice of a deductible in her insurance contract only. In practice, most often she can choose among different levels of a constant deductible. Choosing a deductible in an individual's risk process then corresponds to choosing the deductible in an Excess-ofLoss reinsurance program for an insurance company with a collective risk process. However, in the reinsurance program the insurance company may have other decisions to make, e.g. concerning the proportion covered if only proportional reinsurance is bought on top of the deductible. Typically, an individual does not have such a decision to make. We solve different problems regarding this (lack of) flexibility in the insurance products offered to individuals. Both problems where a constant deductible is chosen (the realistic case) and problems where the deductible is a general function of the loss are solved. Thereby we are able to quantify the welfare loss that arises from giving the individual the choice of a constant deductible only. It is a classical result in non-life insurance that the Excess-of-Loss insurance contract is optimal, so therefore at first glance, there should be no welfare loss. But 
this classical result is only obtained for linear pricing rules. If pricing is non-linear, then the result does not hold anymore and there is, indeed, a welfare loss to detect. We solve the problem for various pricing rules, including non-linear pricing rules.

The objective function in personal financial decision making is often taken to be aggregate utility of consumption financed by wealth, capital gains, and perhaps labor income. This is in contrast to the classical dividend optimization problem for a non-life company where dividends are not bent by a concave utility function before aggregation. On the other hand, dividends run out upon ruin of the insurance company, which in itself forms an indirect aversion towards risk. There exist works where dividends are measured by their utility, but the mainline research counts in dividends linearly in the objective. Apart from the appearance in the objective function with or without a utility function, the consumption of the individual influences individual wealth in the same way as dividends influence the insurance company's risk process. We solve optimal insurance coverage for a general deductible in both cases of exponential and power utility. However, we are able to characterize the solution to the case where the deductible has to be constant in the case of exponential utility only. Therefore, our focus is on this case and the explicit quantification of the welfare loss arising from suboptimal insurance contracts (constant deductible) under non-linear pricing is carried out for exponential utility only. In this type of problem it is possible for the individual to change strategy more often than the time horizon of the objective. It is therefore natural to consider it as a dynamic optimisation problem due to the long-term objective combined with the short-term decisions. This is in contrast to problems where the time horizons for the objective and the decisions are aligned, in which case a one-period (long or short) model suffices. Only with dynamic optimization, future optionality and impact on objectives are correctly counted in and the dependence of state processes time and wealth are revealed. In the versions we consider, time and/or wealth dependence disappear from the optimal controls, but it is important to note that this independence is an endogenous feature of the solution and not exogenously assumed. Generalizations to other cases with dependence should naturally also be based on a dynamic perspective.

The literature on ruin probability minimization and linear dividend optimization until ruin via optimal reinsurance and investment is exhausting. We choose to mention only Schmidli (2002) as well as Schmidli (2008) for an overview. Instead we concentrate here on the somewhat smaller amount of literature where dividends are measured by their utility because the mathematical issues there are more closely related to ours. Hubalek and Schachermayer (2004) considered the problem of optimizing power utility of dividend payments until ruin where the risk process is modelled by a Brownian motion with drift. Their insurance risk process itself is not influenced by the control as it is the case if one optimizes over reinsurance decisions. Grandits et al. (2007) also considered the Brownian risk process but optimized over exponential utility of dividends. However, instead of taking exponential utility of dividend rates they measure exponential utility of aggregate dividends until ruin. Thonhauser and Albrecher (2011) considered the problem of optimizing dividend payouts measured by power utility but with transaction costs related to payouts. Their insurance risk process is not influenced by the control. Common for all these problems is that the dividend payout scheme is the only 
decision process.

It has been argued that the dividend decision process of the insurance company should not be optimized with respect to a utility function as there is not one distinct individual whose utility function can appropriately represent the corporate decision process, see e.g. the survey paper Avanzi et al. (2016). Only from an individual's point of view, the optimization of utility of consumption financed by a wealth process influenced by non-life risk, partly mitigated by the purchase of insurance contracts, appears to be natural. And as such, it could also form the basis for designing insurance contracts. Namely, the optimal insurance contract is the one where adjustments according to characteristics of the individuals (age, wealth, ect.) is an integrated part of the product design, and must be a service provided by the insurance company according to some agreement between the company and the policy holders, in order to make the decision process as simple as possible for them. The worth of considering these issues are quantified by the welfare loss from offering standardized contracts instead of optimal ones. These are the ideas pursued in this paper.

The result that the fixed deductible contract is optimal for linear pricing is found in e.g. Arrow (1971). A series of papers work within this setup of linear pricing. Cummins and Mahul (2004) and Zhou et al. (2010), for example, implement an upper limitation on the insurance coverage of the fixed deductible contract. Golubin (2016) considers instead joint decisions to be made by both the insurance company and the individual. Aase (2017) considers a different approach by arguing how the presence of costs in insurance impacts the design of (Pareto) optimal insurance contracts. This is a certain form of non-linear pricing that also creates a demand for more general deductible structures.

Finally we relate our work to other works where the individual seeks to maximize utility of consumption or wealth from investment and/or non-life insurance decisions. Yang and Zhang (2005) consider the investment problems in a jumpdiffusion model for insurance risk but they control neither consumption nor insurance risk. Moore and Young (2006) study optimal consumption, investment, and insurance under a diffusive financial market and compound Poisson modelled insurance risk. Compared to that, Perera (2010) generalizes both the financial market and the insurance risk model to a general Lévy framework whereas Zou and Cadenillas (2014) generalize to regime shifts in both market and insurance coefficients. Zhang and Siu (2009) control investment and insurance under model uncertainty. Other publications in the area typically generalise the financial market in which an investment decision is made or the preferences of the individual. All the references of this paragraph work with more general financial markets than we do, since we simply earn capital gains from deterministic interest and have no investment decision to make. However, for all the references of this paragraph where insurance is controlled, the pricing mechanism of the insurance market is linear. This makes it optimal, as is shown and used in the references, to buy an insurance product with, in general, a wealth dependent but more importantly in this relation a loss size independent deductible.

The investment decision in financial markets combined with linear insurance pricing in the references mentioned in the prior paragraph marks a clear difference compared to the scope of our work. We concentrate fully on the insurance market, 
look for the optimal insurance position under non-linear pricing, and quantify the financial sacrifice of being offered a flat deductible only. One may argue that since the non-flat deductible is not offered in the retail market, the approach taken by the references is "correct" and our approach is "useless". However, both conclusions are false. It is appealing to think that since retail marketed contracts have flat deductibles, the pricing rule in this market is linear and all other decisions on consumption and investment should be made on that basis. This thinking is based on a blind belief in the market's ability to develop optimal products. If, conversely, the market currently contains suboptimal products only, a couple of important questions arise. Based on the true pricing rules, whatever they are, what is then the optimal insurance decision and how are the consumption and investment decisions altered, respectively, compared to the case of linear pricing? Can the optimal insurance decision inspire to product development with a generalised deductible that actually does represent the market's development of optimal products? And what is the value created to the individuals following from such development of optimal products? These are the type of questions we address in this exposition. So, the motivation is not to repair the decisions made by individuals but rather to repair the market she faces, or at least to start a discussion about whether and why there is something to repair.

In order to start out with explicit and tractable calculations in this direction of study we do make simplifying assumptions on financial markets and preferences as well as we skip considering the investment decision as an integrated problem. This, however, does not harm the principal discussion we start, the qualitative results that we obtain, or the illustrative power of our quantitative results. Whether our analysis suffers more or less from our simplifying assumptions about financial markets and preferences than the analysis in the references above suffer from simplifying assumptions about linear insurance pricing is unknown. But we conjecture that realistic modelling and controlling of financial risk is of second order importance compared to realistic insurance pricing when studying optimal control of insurance risk and optimal design of insurance contracts.

Among the more restrictive assumptions we make, we highlight already here a few. We deal with a marginal problem and not an equilibrium problem. This means that the insurance company does not change the pricing rule (of course, it changes the price itself) depending on decision made by the individual. The alternative, namely to construct a game, would be much more difficult. Further, we consider specific pricing rules. The rules we consider are, however, well-known and generally accepted for their relevance. In the numerical section, the coefficients within the pricing rules are chosen to be realistic, but are not calibrated to any data or price observation, though. Finally, we take the calculations all the way to the end for the case of exponentially distributed losses. This is clearly very restrictive, but this is just to reach fully explicit results in this particular case. We exploit these results in our numerical examples. The assumptions limit our quantitative conclusions to the cases considered. However, they do not limit the outreach of the qualitative discussion about sub-optimality of (realistic) flat deductibles under (realistic) non-linear pricing which is the very motivation for this exposition.

The outline of the paper is as follows. Section 2 explains how insurance affects the individual and her wealth. Section 3 creates a general view of the optimization 
problem of the individual. Section 4 introduces in details the mechanics of pricing by changing measure. Section 5 contains the explicit expressions needed to find the welfare loss for the two insurance products in consideration, namely the one with a fully flexible coverage and the one with a fixed amount deductible. Section 6 makes a numerical comparison by illustrating the welfare loss.

\section{Claims Process and Insurance Contracts}

We consider an individual endowed with the initial wealth $x$. The individual consumes at rate $\left(c_{t}\right)_{t \geq 0}$ and all excess wealth is invested into a risk-free asset with interest rate $r$. The individual is exposed to a risk that can be modelled by a compound Poisson process $\left(A_{t}\right)_{t \geq 0}$ with parameters $(\lambda, F)$, i.e.

$$
A_{t}=\sum_{i=1}^{N_{t}} Z_{i},
$$

where $\left(N_{t}\right)_{t \geq 0}$ is a Poisson process with parameter $\lambda$ counting the number of losses until time $t$ and the $Z_{i}$ 's represent the (positive) loss sizes assumed to be i.i.d. and independent of $\left(N_{t}\right)_{t \geq 0}$ with distribution $F$ on $(0, \infty)$. Note that we speak of losses rather than claims as the analysis is performed on an individual level. The losses $Z_{i}$ are actually the expenses of the individual connected with 'insurable but not yet insured' event number $i$. Thus, it has not become a claim from a policy holder upon an insurance company yet. Before purchasing insurance the wealth of the individual, denoted by $\left(X_{t}\right)_{t \geq 0}$, develops in accordance with

$$
\mathrm{d} X_{t}=\left(r X_{t}-c_{t}\right) \mathrm{d} t-\mathrm{d} A_{t} .
$$

This wealth process of the individual is modelled similarly to a classical surplus process in risk theory with deterministic capital gains and absolutely continuous dividend payments in terms of consumption. It is conventional to think of this as the surplus process for a portfolio of insurances within an insurance company. Here we interpret the process as the wealth process of an individual with event risk modelled by the compound Poisson process.

The individual can reduce and manage the risk of $\left(A_{t}\right)_{t \geq 0}$ by purchasing insurance. An insurance product is described by a non-decreasing function $g_{v}: \mathbb{R}^{+} \rightarrow$ $\mathbb{R}^{+}$parameterized by a control $v$. The function $g_{v}$ is applied to individual losses in the sense that for a claim of size $z, g_{v}(z)$ is the deductible that the individual pays herself. Thus, when choosing the insurance contract $g_{v}$, the expense of the individual is reduced to $\min \left\{z, g_{v}(z)\right\}$ in relation to a loss $z$. When the loss $z>g_{v}(z)$, the loss is reported to the insurance company and the excess $z-g_{v}(z)$ is claimed and covered.

A standard insurance contract has a fixed amount deductible characterized by $g$ being constant, i.e. $g_{K}(z)=K$, where $K \in \mathbb{R}^{+}$is the deductible level that also parametrizes the insurance decision. The individual who we then speak of as a policyholder, reports a claim if the occurred loss $z$ exceeds the fixed amount deductible $K$. So the policyholder covers the loss $\min \{z, K\}$ by herself, and the rest of the loss, namely $(z-K)^{+}$, is covered by the insurance company. Our idea is 
to leave such a restricted class of strategies. The reader may think of a deductible which is more generally dependent on $z$ and, possibly, $t$ and $X_{t}$, such that a variable amount is covered by the insured depending on the size of the claim and, possibly, the time (age) and wealth of the policy holder.

Insurance is used by the policyholder as a tool to reduce her risk exposure $\left(A_{t}\right)_{t \geq 0}$ by reducing the size of the losses. Followed by the above-mentioned arguments, when employing insurance characterized by $g_{v}$, the policy holder's reduced risk can be represented by the compound Poisson process $\left(C_{v, t}\right)_{t \geq 0}$ with jump rate $\lambda$ and claim sizes $\left(\min \left\{Z_{i}, g_{v}\left(Z_{i}\right)\right\}\right)_{i=1,2, \ldots}$, i.e. $C_{v, t}=\sum_{i=1}^{N_{t}} \min \left\{Z_{i}, g_{v}\left(Z_{i}\right)\right\}$. The compound Poisson process $\left(A_{v, t}\right)_{t \geq 0}$ with $A_{v, t}=A_{t}-C_{v, t}=\sum_{i=1}^{N_{t}}\left(Z_{i}-g_{v}\left(Z_{i}\right)\right)^{+}$ then represents the risk transferred to the insurance company for which the policy holder must pay a premium.

Throughout we adopt the idea of evaluating the premium of an insurance contract by a change of measure. Pricing by a change of measure is mostly considered to be a financial notion (also known as risk neutral pricing). Embrechts (2000) provides a treatment of the link between financial pricing and actuarial pricing. We restrict the focus to the equivalent measures $\mathbb{Q}$ such that the accumulated claim process $\left(A_{v, t}\right)_{t \geq 0}$ remains a compound Poisson process under $\mathbb{Q}$, but where the characteristics are altered to $\left(\lambda^{\mathbb{Q}}, F^{\mathbb{Q}}\right)$. We present here how the accumulated premium until time $t$ then can be reduced to a premium density,

$$
\mathbb{E}^{\mathbb{Q}}\left[A_{v, 1}\right]=\mathbb{E}^{\mathbb{Q}}\left[\sum_{i=1}^{N_{1}}\left(Z_{i}-g_{v}\left(Z_{i}\right)\right)^{+}\right]=\lambda^{\mathbb{Q}} \mathbb{E}^{\mathbb{Q}}\left[\left(Z-g_{v}(Z)\right)^{+}\right],
$$

where $Z$ is an independent copy of $Z_{1}, Z_{2}, \ldots$. Since this change of measure is a rather technical concept, we devote Section 4 to elaborate on the needed ingredients. For now, what is important for us is that we can decompose the measure transformation into its jump part, changing the intensity from $\lambda$ to $\lambda^{\mathbb{Q}}$, and claim size part, changing the distribution of $Z$ from $F$ to $F^{\mathbb{Q}}$, due to the compound Poisson properties being maintained under $\mathbb{Q}$. The individual therefore must pay a premium rate continuously that depends on the compound Poisson characteristics $\left(\lambda^{\mathbb{Q}}, F^{\mathbb{Q}}\right)$ under $\mathbb{Q}$, if she wants to buy insurance. In the view of actuarial pricing, this approach is quite general and contains, as special examples, certain versions of the expectation premium principle, a variance premium principle, and an Esscher premium principle, as will be seen in Section 4.

Purchasing insurance then has the following effect on the dynamics of the individual's wealth process

$$
\begin{aligned}
\mathrm{d} X_{t} & =\left(r X_{t}-c_{t}\right) \mathrm{d} t-\mathrm{d}\left(\mathbb{E}^{\mathbb{Q}}\left[A_{v, t}\right]\right)-\mathrm{d} C_{v, t} \\
& =\left(r X_{t}-c_{t}-\lambda^{\mathbb{Q}} \mathbb{E}^{\mathbb{Q}}\left[\left(Z-g_{v}(Z)\right)^{+}\right]\right) \mathrm{d} t-\mathrm{d} C_{v, t} .
\end{aligned}
$$

With these dynamics, the infinitesimal operator of a function $f(x)$ is

$$
\begin{aligned}
\mathcal{A} f=\left(r x-c_{t}-\lambda \mathbb{Q}_{\mathbb{E}} \mathbb{Q}[\right. & \left.\left.\left(Z-g_{v}(Z)\right)^{+}\right]\right) \frac{\partial f}{\partial x}(x) \\
& +\lambda \mathbb{E}\left[f\left(x-\min \left\{Z, g_{v}(Z)\right\}\right)-f(x)\right] .
\end{aligned}
$$




\section{Optimization problem of the individual}

At time $t$ the individual chooses the consumption rate and the insurance strategy in terms of $g_{v}$ in order to optimize her expected discounted utility of consumption,

$$
V(t, x)=\sup _{c, v} \mathbb{E}_{t, x}\left[\int_{t}^{\infty} \exp (-\rho(s-t)) u\left(c_{s}\right) \mathrm{d} s\right],
$$

where $\mathbb{E}_{t, x}$ denotes conditional expectation given that $X_{t}=x$. The utility function $u$ measures utility from the consumption rate $c$ and $\rho$ is a subjective utility discount factor. We do not impose any control constraints on consumption. A natural (and common) restriction would be $c \geq 0$, but as we prioritize finding a tractable solution, we choose to look past this. The insurance control $v$, on the other hand, must satisfy that the deductible strategy $g_{v}$ is non-decreasing and non-negative (as it was defined). We only put a lower limit on the deductible strategy as we avoid any issues with upper limitations by using truncation and minimum in the dynamics of the wealth (2). Hence, if a deductible strategy exceeds the actual loss, then this automatically corresponds to having no insurance.

Due to the time-homogeneity of all ingredients of the state process, i.e. the coefficients of $X$, and the objective, i.e. $(\rho, u)$, the value function is a function of wealth only and we can write

$$
V(x)=\sup _{c, v} \mathbb{E}_{x}\left[\int_{0}^{\infty} \exp (-\rho t) u\left(c_{t}\right) \mathrm{d} t\right] .
$$

The rate $\rho$ is here called the utility discount rate. A different interpretation is the mortality rate of an individual optimizing her utility of consumption until death. Then, if an expectation is taken both with respect to time of death and insurance risk, the expectation with respect to time of death gives a survival probability until time $t$ of $\exp (-\rho t)$ and expectation with respect to both risks then leads to (4). It is of course a non-realistic restriction, in that interpretation, to work with an age-independent mortality rate. However, for now we work out the details for the time-homogeneous case and it is beyond the scope of this presentation to handle the time-inhomogeneous case. The Hamilton-Jacobi-Bellman (HJB) equation characterizing the value function is given by

$$
\sup _{c, v}\{\mathcal{A} V+u(c)\}=\rho V(x) .
$$

Using the infinitesimal operator in (3) the HJB equation can be written more explicitly as

$$
\begin{aligned}
\sup _{c, v}\{-\rho V(x)+( & \left.r x-c-\lambda^{\mathbb{Q}} \mathbb{E}^{\mathbb{Q}}\left[\left(Z-g_{v}(Z)\right)^{+}\right]\right) V_{x}(x) \\
& \left.+\lambda \mathbb{E}\left[V\left(x-\min \left\{g_{v}(Z), Z\right\}\right)-V(x)\right]+u(c)\right\}=0,
\end{aligned}
$$

where $V_{x}(x)=\frac{\partial V}{\partial x}(x)$.

For the special case of an exponential utility function, we can immediately learn something about the structure of $V$. Since this case plays a crucial role in our considerations we present at this point these principal observations. 
Proposition 3.1. Assume that the utility function is of the form

$$
u(c)=\frac{-1}{a} \exp (-a c) .
$$

for $a>0$. Then, for a sufficiently regular function $g_{v}(z)$ (in both $z$ and the parameter $v$ ), the value function (4) can be written by

$$
V(x)=\frac{-1}{\alpha} \exp (-\operatorname{rax}) .
$$

The optimal consumption $c^{*}$ is affine in wealth

$$
c^{*}=r x-\frac{1}{a} \log \left(\frac{r a}{\alpha}\right),
$$

and the optimal insurance control $v^{*}$ solves

$$
\lambda \frac{\partial}{\partial v}\left(\mathbb{E}\left[\exp \left(r a \cdot \min \left\{Z, g_{v}(Z)\right\}\right)\right]\right)=r a \lambda^{\mathbb{Q}} \frac{\partial}{\partial v}\left(\mathbb{E}^{\mathbb{Q}}\left[\left(Z-g_{v}(Z)\right)^{+}\right]\right),
$$

and is thus independent of wealth. The parameter $\alpha$ of the value function is determined by the relation

$$
\alpha=r a \exp \left(\begin{array}{c}
\frac{1}{r}\left(\rho-\lambda\left(\mathbb{E}\left[\exp \left(r a \cdot \min \left\{Z, g_{v^{*}}(Z)\right\}\right)\right]-1\right)\right) \\
-a \mathbb{Q}_{\mathbb{E}} \mathbb{Q}\left[\left(Z-g_{v^{*}}(Z)\right)^{+}\right]-1
\end{array}\right) .
$$

Proof. Consider the first order conditions of (5) with respect to consumption

$$
-V_{x}(x)+\exp (-a c)=0,
$$

and with respect to deductible

$$
\left.-\frac{\partial}{\partial v}\left(\lambda^{\mathbb{Q}} \mathbb{E}^{\mathbb{Q}}\left[\left(Z-g_{v}(Z)\right)^{+}\right]\right) V_{x}(x)+\lambda \mathbb{E}\left[V\left(x-\min \left\{Z, g_{v}(Z)\right\}\right)\right]\right)=0 .
$$

Note that in the case where the parameter $v$ is multidimensional, the derivative would be replaced by the gradient, which would yield the same number of first order conditions as dimensions of $v$.

Conjecture that the solution to the HJB equation is of type $V(x)=-\exp (-r a$. $x) / \alpha$. The first order condition with respect to the consumption is then

$$
-\frac{r a}{\alpha} \exp (-r a \cdot x)+\exp (-a c)=0 .
$$

Reducing this leads to the optimal consumption

$$
c^{*}=r x-\frac{1}{a} \log \left(\frac{r a}{\alpha}\right),
$$

Correspondingly for the first order condition with regards to the insurance control,

$$
\begin{aligned}
-\lambda^{\mathbb{Q}} \frac{\partial}{\partial v} & \left(\mathbb{E}^{\mathbb{Q}}\left[\left(Z-g_{v}(Z)\right)^{+}\right]\right) \frac{r a}{\alpha} \exp (-r a \cdot x) \\
& +\frac{\lambda}{\alpha} \frac{\partial}{\partial v}\left(\mathbb{E}\left[\exp \left(-r a \cdot\left(x-\min \left\{Z, g_{v}(Z)\right\}\right)\right)\right]\right)=0
\end{aligned}
$$


and the optimal deductible strategy, $v^{*}$, must therefore be a solution to

$$
\lambda \frac{\partial}{\partial v}\left(\mathbb{E}\left[\exp \left(r a \cdot \min \left\{Z, g_{v}(Z)\right\}\right)\right]\right)=r a \lambda^{\mathbb{Q}} \frac{\partial}{\partial v}\left(\mathbb{E}^{\mathbb{Q}}\left[\left(Z-g_{v}(Z)\right)^{+}\right]\right) .
$$

Although not solved explicitly, it is clear that the first order condition for the deductible strategy does not depend on wealth. Regularity of $g_{v}(z)$ is assumed in the proposition to ensure existence and uniqueness of (9). Exact sufficient and necessary conditions are not studied here.

When substituting these optimal values back into the HJB equation (5), the supremum will be obtained and we can solve for (10), verifying that the initial guess for the structure of the value function was correct, since $\alpha$ does not depend on wealth.

From (8) we learn that the individual stops consuming, $c^{*} \rightarrow 0$, when the wealth approaches some lower level, $x \rightarrow \log (r a / \alpha) / r a$. Only the insurable losses will therefore cause the wealth to decrease below this level yielding negative consumption. In relation to this, it is natural to point out that the wealth is allowed to become negative also, but the controlled wealth grows (on average) linearly in time since the optimal insurance control is independent of wealth and the optimal consumption is linear in wealth with a factor equal to the risk free interest rate. Hence, over-accumulation of wealth or long-term bankruptcy is not an issue here. It can not be verified in general that the insurance control chosen by (9) satisfies that $g_{v^{*}}$ is non-decreasing and non-negative, so this must be taken into consideration for the specific structures of $g_{v}$ and pricing measures $\mathbb{Q}$.

We are going to compare the performance of the optimal decisions $\left(c^{*}, v^{*}\right)$ with alternative decisions, in particular with suboptimal choices of $v$. For this purpose we characterize the solution to the problem (4) where supremum is taken over $c$ only, i.e.

$$
V(x)=\sup _{c} \mathbb{E}_{x}\left[\int_{0}^{\infty} \exp (-\rho t) u\left(c_{t}\right) \mathrm{d} t\right],
$$

such that the value function is characterized by the HJB equation

$$
\sup _{c}\{\mathcal{A} V+u(c)\}=\rho V(x) .
$$

Going through the relevant steps in the proof of Proposition 3.1, one can immediately see that the structure of the solution in the exponential utility case is preserved. The value function has again the structure (7) with a different $\alpha$ determined by (10) with $v^{*}$ replaced by a given suboptimal deductible. It should be mentioned that any disentanglement of the optimization over $c$ and $v$ is of course true only if the deductible does not depend on wealth. Dependence on wealth, in case of other utility functions, introduces interdependence between consumption and insurance decisions.

We can now compare a suboptimal insurance decision with the optimal one by comparing the two value functions arising from optimizing over $(c, v)$ and from optimizing over $c$ for a given suboptimal $v$, respectively. Let us denote by $V^{(1)}$ the value function arising from optimizing over $(c, v)$ giving rise to the coefficient $\alpha_{1}$ and denote by $V^{(2)}$ the value function arising from optimizing over $c$ for a given 
$v$ giving rise to the coefficient $\alpha_{2}$. It is clear that $V^{(1)} \geq V^{(2)}$ and $\alpha_{1} \geq \alpha_{2}$, since the optimal insurance decision beats the suboptimal one. In order to compare, it is standard to transform the difference into monetary units by calculating the socalled certainty equivalent of the loss of utility from implementing the suboptimal insurance decision. This is the solution $L$ to the utility indifference equality

$$
V^{(1)}(x-L)=V^{(2)}(x) .
$$

The idea of utility indifference is a generally accepted way to measure sub-optimality. One may first think of calculating $V^{(1)}(x)-V^{(2)}(x)$ as a measure of sub-optimality. However, the nominal value of the value function does not have a meaning on its own and therefore this difference contains no other information than which one is preferred to the other due to its sign. But how should one then measure e.g. significance of the difference? This is exactly what the utility indifference equality does since when the difference between the value functions is 0 , then this nominal value has a clear economic interpretation of the indifference. Thus, the equality translates the difference between value functions, which is non-informative, into an informative quantity in monetary units. It answers the question, what is the loss in monetary units from having access to only a suboptimal control compared to being offered the optimal control?

It is immediately seen that for the exponential utility function, the solution is

$$
L=\frac{1}{r a} \log \left(\frac{\alpha_{1}}{\alpha_{2}}\right) .
$$

We are ultimately interested in determining this $L$ for different suboptimal choices of $v$, for different pricing measures $\mathbb{Q}$, and for different distributions of $Z$ in order to get a general view on the loss of not having access to the optimal insurance contract.

\section{Pricing by change of measure}

As briefly explained in connection to (1), we want to price insurance by a change of measure. In a risk averse setting, the change of measure has the advantage that one can give more weight to bad outcomes and hence assign a higher price to larger risks. A simple example on how this affects the individual's attitude towards insurance coverage is presented in Appendix A.

Let the physical measure be denoted as $\mathbb{P}$. Recall that under this measure the characteristics of the compound Poisson risk process $\left(A_{t}\right)_{t \geq 0}$ can be summarized by $(\lambda, F)$. Let $\beta: \mathbb{R}^{+} \rightarrow \mathbb{R}$ be a Borel measurable mapping, henceforth referred to as the pricing measure function, satisfying $\mathbb{E}[\exp (\beta(Z))]<\infty$. Delbaen and Haezendonck (1989) define the Radon-Nikodym derivative

$$
M_{t}^{\beta}=\exp \left(\sum_{i=1}^{N_{t}} \beta\left(Z_{i}\right)-\lambda t \mathbb{E}[\exp (\beta(Z))-1]\right) .
$$

Let $\left(\mathcal{F}_{t}\right)_{t \geq 0}$ denote the filtration generated by the compound Poisson process $\left(A_{t}\right)_{t \geq 0}$. Delbaen and Haezendonck (1989) argues that the measure $\mathbb{Q}(\beta)$ defined by $M_{t}^{\beta}=\mathbb{E}\left[\mathrm{d} \mathbb{Q}(\beta) / \mathrm{d} \mathbb{P} \mid \mathcal{F}_{t}\right]$ satisfies that 
- $\mathbb{Q}(\beta)$ and $\mathbb{P}$ are progressively equivalent, i.e. have the same null sets.

- $\left(A_{t}\right)_{t \geq 0}$ is a $\mathbb{Q}(\beta)$-compound Poisson process with characteristics $\left(\lambda^{\mathbb{Q}(\beta)}, F^{\mathbb{Q}(\beta)}\right)$ given by

$$
\lambda^{\mathbb{Q}(\beta)}=\lambda \mathbb{E}[\exp (\beta(Z))], \quad F^{\mathbb{Q}(\beta)}(\mathrm{d} z)=\frac{\exp (\beta(z))}{\mathbb{E}[\exp (\beta(Z))]} F(\mathrm{~d} z) .
$$

So $\mathbb{E}[\exp (\beta(Z))]$ can be considered as a penalty for claim frequency risk and $\exp (\beta(z)) / \mathbb{E}[\exp (\beta(Z))]$ as a penalty for claim size risk.

A premium rate of the risk $\left(A_{t}\right)_{t \geq 0}$ can then be defined as

$$
\mathbb{E}^{\mathbb{Q}(\beta)}\left[A_{1}\right]=\mathbb{E}^{\mathbb{Q}(\beta)}\left[\sum_{i=1}^{N_{1}} Z_{i}\right]=\lambda \mathbb{E}[\exp (\beta(Z)) Z],
$$

where we take the expected value under the pricing measure $\mathbb{Q}(\beta)$ which maintains the compound Poisson structure, but changes the claim frequency and claim size distribution (cf. the second property above).

In Delbaen and Haezendonck (1989) it is argued how certain choices of $\beta(\cdot)$ correspond to certain well-known premium principles. Three choices of the pricing measure function are considered. We recapitulate briefly the findings here.

i. Constant: $\beta(z)=\delta$. The premium rate (16) then reduces to

$$
\mathbb{E}^{\mathbb{Q}(\beta)}\left[A_{1}\right]=\lambda \exp (\delta) \mathbb{E}[Z] .
$$

Only claim frequency risk is penalized, whereas claim size risk is not priced. Since the pricing measure function is constant, the price is linear in the expectation under $\mathbb{P}$, and we are in the case of the expected value premium principle. This corresponds to the case exemplified also at the end of Section A with

$$
\lambda^{\mathbb{Q}}=\lambda \exp (\delta) .
$$

ii. Log-linear: $\beta(z)=\log (\theta z+\delta)$. If $\delta=1-\theta \mathbb{E}[Z]>0$ implicating $\mathbb{E}[\exp (\beta(Z))]=$ 1 , then it appears from (16) that

$$
\mathbb{E}^{\mathbb{Q}(\beta)}\left[A_{1}\right]=\lambda(\mathbb{E}[Z]+\theta \mathbb{V}[Z]) .
$$

This is the reversed situation compared to i), since here the claim frequency risk is not priced whereas claim size risk is priced using the variance premium principle. If $\delta=1$, the premium principle corresponds to the variance principle used on the total claim amount since

$$
\mathbb{E}^{\mathbb{Q}(\beta)}\left[A_{t}\right]=\lambda t \mathbb{E}\left[\left(\theta Z^{2}+Z\right)\right]=\mathbb{E}\left[A_{t}\right]+\theta \mathbb{V}\left[A_{t}\right] .
$$

iii. Linear: $\beta(z)=\theta z+\delta$. If $\delta=-\log (\mathbb{E}[\exp (\theta Z)])$ it once again implicates that $\mathbb{E}[\exp (\beta(Z))]=1$. From (16) it follows that

$$
\mathbb{E}^{\mathbb{Q}(\beta)}\left[A_{1}\right]=\lambda \frac{\mathbb{E}[Z \exp (\theta Z)]}{\mathbb{E}[\exp (\theta Z)]} .
$$

As in ii) claim frequency risk has no penalty, and the claim sizes are priced according to the Esscher premium principle. 
In Delbaen and Haezendonck (1989), the variable $Z_{i}$ is the claim on the insurance company from insurance event number $i$. Recall that here, $Z_{i}$ is the true loss whereas only a part of this is claimed on the insurance company. The individual does not necessarily buy insurance protection for the entire underlying risk $\left(A_{t}\right)_{t \geq 0}$. Instead, insurance splits the losses, and thus the risk, in two parts, one covered by the policy holder $\left(C_{v, t}\right)_{t \geq 0}$ and one by the insurance company $\left(A_{v, t}\right)_{t \geq 0}$, when the insurance product is characterized by $g_{v}$. If the same approach as in Delbaen and Haezendonck (1989) was directly followed, then the premium of the partial risk $\left(A_{v, t}\right)_{t \geq 0}$ would have to be altered as follows,

$$
\mathbb{E}^{\mathbb{Q}\left(\beta_{v}\right)}\left[A_{v, 1}\right]=\mathbb{E}^{\mathbb{Q}\left(\beta_{v}\right)}\left[\sum_{i=1}^{N_{1}}\left(Z_{i}-g_{v}\left(Z_{i}\right)\right)^{+}\right]=\lambda \mathbb{E}\left[\exp \left(\beta_{v}(Z)\right)\left(Z-g_{v}(Z)\right)^{+}\right],
$$

where $\beta_{v}(z)=\beta\left(\left(z-g_{v}(z)\right)^{+}\right)$. Note that the insurance control $v$ then appears as an endogenous part of the pricing measure function. This considerably complicates the first order condition (9) for the insurance control in the policy holder's optimization problem. The result would be a kind of equilibrium insurance strategy. We wish to work within a marginal approach where we separate the pricing measure function from the control problem by making it independent of the insurance control $v$. We use the change of measure defined by (14) applied to the full claim to price the partial risk covered by the insurance company. The following proposition verifies that this is still a meaningful change of measure.

Proposition 4.1. Let compound Poisson characteristics of $\left(A_{v, t}\right)_{t \geq 0}$ be summarized by $\left(\lambda, F_{v}\right)$. Under $\mathbb{Q}(\beta)$ the process $\left(A_{v, t}\right)_{t \geq 0}$ is still a compound Poisson process, but with altered characteristics $\left(\lambda^{\mathbb{Q}(\beta)}, F_{v}^{\mathbb{Q}(\beta)}\right)$ where

$$
\lambda^{\mathbb{Q}(\beta)}=\lambda \mathbb{E}[\exp (\beta(Z))], \quad F_{v}^{\mathbb{Q}(\beta)}(d z)=\frac{\exp (\beta(z))}{\mathbb{E}[\exp (\beta(Z))]} F_{v}(d z) .
$$

The premium rate is

$$
\mathbb{E}^{\mathbb{Q}(\beta)}\left[A_{v, 1}\right]=\lambda \mathbb{E}\left[\exp (\beta(Z))\left(Z-g_{v}(Z)\right)^{+}\right] .
$$

The proof appears in Appendix B. The intuition is that even though claim sizes have been transformed by $h_{v}$, it is the change of characteristics of the underlying risk $\left(A_{t}\right)_{t \geq 0}$ that is determined by $\mathbb{Q}(\beta)$. The same choices i.-iii. of $\beta$ are considered in the following sections, but with different restrictions on the parameters. In general, we are interested in parameters $\delta$ and $\theta$ such that $\beta$ is non-decreasing and positive, hence we consider the pricing measure functions

i. $\beta_{1}(z)=\delta_{1}$, where $\delta_{1} \in \mathbb{R}^{+}$.

ii. $\beta_{2}(z)=\log \left(\theta_{2} z+\delta_{2}\right)$, where $\left(\theta_{2}, \delta_{2}\right) \in \mathbb{R}^{+} \times[1, \infty)$.

iii. $\beta_{3}(z)=\theta_{3} z+\delta_{3}$, where $\left(\theta_{3}, \delta_{3}\right) \in \mathbb{R}^{+} \times \mathbb{R}^{+}$.

Further restrictions on the parameter values might be imposed in subsequent analysis when necessary. Note that the link from (16) to various premium principles does not hold for (18) though, since the change of measure no longer relates to the claim size but the entire loss. 


\section{Insurance products}

The results in Section A show that a constant deductible is not optimal in the case of non-linear pricing, i.e. pricing where $\lambda_{i}^{\mathbb{Q}} / \lambda_{i}$ is not constant in $i$. This means that there is a welfare loss connected with having access to only constant deductibles in the market. The welfare loss depends, of course, on the extent and shape of the non-linearity. In section 4 we have introduced a family of non-linear pricing rules, and we are now going to measure the welfare loss produced by these pricing rules. The welfare loss is measured by comparing the optimal insurance design corresponding to its full flexibility in the structure of the deductible, with the suboptimal constant deductible insurance contract corresponding to no flexibility in the structure of the deductible. To compare these, we use the loss (13), which also can be written as

$$
\begin{gathered}
L=\frac{1}{r a}\left(a \lambda\left(\mathbb{E}\left[\exp (\beta(Z))\left(Z-g_{v}^{(2)}(Z)\right)^{+}\right]-\mathbb{E}\left[\exp (\beta(Z))\left(Z-g_{v}^{(1)}(Z)\right)^{+}\right]\right)\right. \\
\left.+\frac{\lambda}{r}\left(\mathbb{E}\left[\exp \left(r a \min \left\{Z, g_{v}^{(2)}(Z)\right\}\right)\right]-\mathbb{E}\left[\exp \left(r a \min \left\{Z, g_{v}^{(1)}(Z)\right\}\right)\right]\right)\right)
\end{gathered}
$$

Hence for two insurance products (full and no flexibility) and for each pricing measure function $\beta(\cdot)$ we need to calculate the terms

$$
\begin{aligned}
& p_{\beta}^{(i)}=\mathbb{E}\left[\exp (\beta(Z))\left(Z-g_{v}^{(i)}(Z)\right)^{+}\right] \\
&=\mathbb{P}\left(Z>g_{v}^{(i)}(Z)\right) \mathbb{E}\left[\exp (\beta(Z))\left(Z-g_{v}^{(i)}(Z)\right) \mid Z>g_{v}^{(i)}(Z)\right], \\
& q_{\beta}^{(i)}=\mathbb{E}\left[\exp \left(r a \min \left\{Z, g_{v}^{(i)}(Z)\right\}\right)\right] \\
&=\mathbb{P}\left(Z \leq g_{v}^{(i)}(Z)\right) \mathbb{E}\left[\exp (r a Z) \mid Z \leq g_{v}^{(i)}(Z)\right] \\
& \quad+\mathbb{P}\left(Z>g_{v}^{(i)}(Z)\right) \mathbb{E}\left[\exp \left(r a g_{v}^{(i)}(Z)\right) \mid Z>g_{v}^{(i)}(Z)\right] .
\end{aligned}
$$

for $i=1,2$. Recall that $i=1$ corresponds to the completely flexible insurance product, where the deductible strategy is unparameterized, i.e. $g_{v}^{(1)}(z)=g(z)$, and $i=2$ corresponds to the fixed amount deductible insurance product, hence $g_{v}^{(2)}(z)=K$.

The aim of this section is not to obtain a closed form expression of welfare loss $L$, but to find the ingredients (19) for the different $\beta$-functions of interest. The welfare loss is then illustrated by an numerical example in the next section. The relations in (19) depend indeed on the distribution of the claim sizes. In order to obtain tractable expressions, we assume that the claim sizes are exponentially distributed with parameter $\eta$, i.e. $F(z)=1-\exp (-\eta z)$. Only the results will be stated here, for calculations of this section see appendix.

\subsection{Completely flexible}

Assume that the individual can choose a deductible strategy freely as a function of the loss. She is then facing the problem of solving

$$
\begin{aligned}
\sup _{c, g(\cdot)}\{-\rho V(x) & +\left(r x-c-\lambda \mathbb{E}\left[\exp (\beta(Z))(Z-g(Z))^{+}\right]\right) V_{x}(x) \\
& +\lambda(\mathbb{E}[V(x-\min \{Z, g(Z)\})]-V(x))+u(c)\}=0 .
\end{aligned}
$$


The first order condition with respect to $g(\cdot)$ is

$$
\lambda \mathbb{E}\left[\exp (\beta(Z)) \mathbb{1}_{Z \geq g(Z)}\right] V_{x}(x)=\lambda \mathbb{E}\left[V_{x}(x-g(Z)) \mathbb{1}_{Z \geq g(Z)}\right] .
$$

Recall that the value function has the form $V(x)=\exp (-\operatorname{rax}) / \alpha$ for an appropriate $\alpha$, hence the optimal unrestricted insurance strategy, $g^{*}(\cdot)$, must satisfy

$$
\mathbb{E}\left[\exp (\beta(Z)) \mathbb{1}_{Z \geq g(Z)}\right]=\mathbb{E}\left[\exp (r a \cdot g(Z)) \mathbb{1}_{Z \geq g(Z)}\right] .
$$

These moments are matched when $g^{*}(z)=\beta(z) /(r a)$. We consider the three choices of the pricing measure function $\beta$ introduced in the end of Section 5 . The index of $\beta$ is reflected in the index of the optimal insurance strategy $g^{*}$. Remember that $g$ is unparameterized in this section, so subscripts of the optimal strategy $g^{*}$ refer to the pricing measure function and not the parameterization of $g$.

i. For a constant pricing measure function $\beta_{1}(z)=\delta_{1}$, the optimal insurance strategy for the individual is the fixed amount deductible, $g_{1}^{*}(z)=\delta_{1} /(r a)$. This was anticipated due to the preliminary analysis of Section A. Let $A_{1}=$ $\left\{Z<\delta_{1} /(\mathrm{ra})\right\}$. The two insurance dependent terms (19) of $L$ can then be written as

$$
\begin{gathered}
p_{\beta_{1}}^{(2)}=\mathbb{E}\left[\exp \left(r a \cdot \min \left\{Z, g_{1}^{*}(Z)\right\}\right)\right]=\mathbb{E}\left[\exp \left(r a \cdot \min \left\{Z, \delta_{1} /(r a)\right\}\right)\right] \\
=\mathbb{P}\left(A_{1}\right) \mathbb{E}\left[\exp (r a Z) \mid A_{1}\right]+\mathbb{P}\left(A_{1}^{c}\right) \exp \left(\delta_{1}\right), \\
q_{\beta_{1}}^{(2)}=\mathbb{E}\left[\exp \left(\beta_{1}(Z)\right)\left(Z-g_{1}^{*}(Z)\right)^{+}\right]=\mathbb{E}\left[\exp \left(\delta_{1}\right)\left(Z-\delta_{1} /(r a)\right)^{+}\right] \\
=\mathbb{P}\left(A_{1}^{c}\right) \exp \left(\delta_{1}\right) \mathbb{E}\left[Z-\delta_{1} /(r a) \mid A_{1}^{c}\right] .
\end{gathered}
$$

Assuming that $Z$ is exponentially distributed with parameter $\eta$, then we can write these more explicitly as

$$
\begin{aligned}
p_{\beta_{1}}^{(2)} & =\frac{r a}{r a-\eta} \exp \left(\left(\frac{r a-\eta}{r a}\right) \delta_{1}\right)-\frac{\eta}{r a-\eta}, \\
q_{\beta_{1}}^{(2)} & =\frac{1}{\eta} \exp \left(\left(\frac{r a-\eta}{r a}\right) \delta_{1}\right) .
\end{aligned}
$$

ii. If the pricing measure function is $\log$-linear, $\beta_{2}(z)=\log \left(\theta_{2} z+\delta_{2}\right)$, then the optimal insurance strategy is a logarithmic-power deductible, $g_{2}^{*}(z)=\log \left(\theta_{2} z+\right.$ $\left.\delta_{2}\right) /(r a)$. Let $A_{2}=\left\{Z<\log \left(\theta_{2} Z+\delta_{2}\right) /(r a)\right\}$. The terms (19) are then defined by

$$
\begin{gathered}
p_{\beta_{2}}^{(2)}=\mathbb{E}\left[\exp \left(r a \cdot \min \left\{Z, g_{2}^{*}(Z)\right\}\right)\right]=\mathbb{E}\left[\exp \left(r a \cdot \min \left\{Z, \log \left(\theta_{2} Z+\delta_{2}\right) /(r a)\right\}\right)\right] \\
=\mathbb{P}\left(A_{2}\right) \mathbb{E}\left[\exp (r a Z) \mid A_{2}\right]+\mathbb{P}\left(A_{2}^{c}\right) \mathbb{E}\left[\exp \left(\theta Z+\delta_{2}\right) \mid A_{2}^{c}\right], \\
q_{\beta_{2}}^{(2)}=\mathbb{E}\left[\exp \left(\beta_{2}(Z)\right)\left(Z-g_{2}^{*}(Z)\right)^{+}\right]=\mathbb{E}\left[\left(\theta_{2} Z+\delta_{2}\right)\left(Z-\log \left(\theta_{2} Z+\delta_{2}\right) /(r a)\right)^{+}\right] \\
=\mathbb{P}\left(A_{2}\right) \mathbb{E}\left[\left(\theta_{2} Z+\delta_{2}\right)\left(Z-\log \left(\theta_{2} Z+\delta_{2}\right) /(r a)\right) \mid A_{2}^{c}\right] .
\end{gathered}
$$

The challenge here is to evaluate the event $A_{2}$. Calculations in Appendix D show that

$$
A_{2}=\{Z<Q\} \text { where } Q=-\frac{1}{r a} W_{-1}\left(-\frac{r a}{\theta_{2}} \exp \left(-\delta_{2} \frac{r a}{\theta_{2}}\right)\right)-\frac{\delta_{2}}{\theta_{2}} .
$$


using the lower branch $W_{-1}$ of the Lambert $W$ function defined as the inverse of the function $w \mapsto w \exp (w)$.

For an exponential distribution of the claim sizes, we then have

$$
\mathbb{P}\left(A_{2}\right)=\mathbb{P}(Z<Q)=1-\exp (-\eta Q),
$$

which yields

$$
\begin{gathered}
p_{\beta_{2}}^{(2)}=\frac{\eta}{r a-\eta}(\exp ((r a-\eta) Q)-1)+\left(\theta_{2} Q+\delta_{2}+\frac{\theta_{2}}{\eta}\right) \exp (-\eta Q), \\
q_{\beta_{2}}^{(2)}=\exp (-\eta Q)\left(\theta_{2} Q^{2}+\left(\delta_{2}+2 \frac{\theta_{2}}{\eta}\right) Q-\frac{1}{\eta}\left(\theta_{2}-\delta_{2}\right)+2 \frac{\theta_{2}}{\eta^{2}}\right. \\
-\frac{1}{r a} \frac{\theta_{2}}{\eta} \exp \left(\frac{\eta}{\theta_{2}}\left(\theta_{2} Q+\delta_{2}\right)\right) E_{1}\left(\frac{\eta}{\theta_{2}}\left(\theta_{2} Q+\delta_{2}\right)\right) \\
\left.-\frac{1}{r a}\left(\theta_{2} Q+\delta_{2}+\frac{\theta_{2}}{\eta}\right) \log \left(\theta_{2} Q+\delta_{2}\right)\right)
\end{gathered}
$$

where $E_{1}$ denotes the exponential integral $E_{1}(x)=\int_{x}^{\infty} \exp (-t) / t \mathrm{~d} t$.

iii. Employing a linear pricing measure function $\beta_{3}(z)=\theta_{3} z+\delta_{3}$, then the individual optimally chooses a proportional insurance strategy $g_{3}^{*}(z)=\left(\theta_{3} z+\delta_{3}\right) /(\mathrm{ra})$. Letting $A_{3}=\left\{Z<\left(\theta_{3} Z+\delta_{3}\right) /(r a)\right\}=\left\{Z<\delta_{3} /\left(r a-\theta_{3}\right)\right\}$, then we have

$$
\begin{gathered}
p_{\beta_{3}}^{(2)}=\mathbb{E}\left[\exp \left(r a \cdot \min \left\{Z, g_{3}^{*}(Z)\right\}\right)\right]=\mathbb{E}[\exp (r a \cdot \min \{Z,(\theta Z+\delta) /(r a)\})] \\
=\mathbb{P}\left(A_{3}\right) \mathbb{E}\left[\exp (r a Z) \mid A_{3}\right]+\mathbb{P}\left(A_{3}^{c}\right) \mathbb{E}\left[\exp \left(\theta_{3} Z+\delta_{3}\right) \mid A_{3}^{c}\right], \\
q_{\beta_{3}}^{(2)}=\mathbb{E}\left[\exp \left(\beta_{3}(Z)\right)\left(Z-g_{3}^{*}(Z)\right)^{+}\right]=\mathbb{E}\left[\exp \left(\theta_{3} Z+\delta_{3}\right)\left(Z-\left(\theta_{3} Z+\delta_{3}\right) /(r a)\right)^{+}\right] \\
=\mathbb{P}\left(A_{3}^{c}\right) \mathbb{E}\left[\exp \left(\theta_{3} Z+\delta_{3}\right)\left(Z-\left(\theta_{3} Z+\delta_{3}\right) /(r a)\right) \mid A_{3}^{c}\right] .
\end{gathered}
$$

For an exponential distribution we can calculate these explicitly as

$$
\begin{aligned}
& p_{\beta_{3}}^{(2)}=(1-\exp (\left.\left.-(\eta-r a) \frac{\delta_{3}}{r a-\theta_{3}}\right)\right)\left(\frac{\eta}{\eta-r a}\right) \\
&+\frac{\eta}{\eta-\theta_{3}} \exp \left(-(\eta-r a) \frac{\delta_{3}}{r a-\theta_{3}}\right), \\
& q_{\beta_{3}}^{(2)}=\exp \left(-(\eta-r a) \frac{\delta_{3}}{r a-\theta_{3}}\right) \frac{\left(r a-\theta_{3}\right) \eta}{r a\left(\eta-\theta_{3}\right)^{2}},
\end{aligned}
$$

where it is assumed that $\theta_{3}<\eta$ to ensure finite (left-truncated) exponential moments, and $\theta_{3}<r a$ in order for $A_{3}^{c}$ not to be a null set.

\subsection{One level fixed amount deductible}

Consider the fixed amount deductible where $g_{K}(z)=K$ is constant. The HJB equation is then

$$
\begin{aligned}
\sup _{c, K}\{-\rho V(x) & +\left(r x-c-\lambda \mathbb{E}\left[\exp (\beta(Z))(Z-K)^{+}\right]\right) V_{x}(x) \\
& +\lambda(\mathbb{E}[V(x-\min \{Z, K\})]-V(x))+u(c)\}=0 .
\end{aligned}
$$


The first order condition for the fixed amount deductible level follows from using Leibniz integral rule on (9) yielding

$$
\mathbb{E}\left[\exp (\beta(Z)) \mathbb{1}_{\{Z \geq K\}}\right]=\exp (r a K) \mathbb{E}\left[\mathbb{1}_{\{Z \geq K\}}\right] .
$$

Once again, we take a closer look at the three choices of the pricing measure function. Note that since it is the same insurance product in question, the second characterizations in (19), namely

$$
\begin{aligned}
q_{\beta}^{(1)} & =\mathbb{E}[\exp (r a \min \{Z, K\})] \\
& =\mathbb{P}(Z \leq K) \mathbb{E}[\exp (r a Z) \mid Z \leq K]+\mathbb{P}(Z>K) \exp (\text { raK }),
\end{aligned}
$$

is the same function of the fixed deductible in every case of the pricing measure function. For an exponential distribution it can be calculated as in case i. for the flexible insurance product,

$$
q_{\beta}^{(1)}=\frac{r a}{r a-\eta} \exp ((r a-\eta) K)-\frac{\eta}{r a-\eta} .
$$

So the focus in the following is on characterizing

$$
p_{\beta}^{(1)}=\mathbb{E}\left[\exp (\beta(Z))(Z-K)^{+}\right]=\mathbb{P}(Z>K) \mathbb{E}[\exp (\beta(Z))(Z-K) \mid Z>K] .
$$

i. For $\beta_{1}(z)=\delta_{1}$, the optimal deductible is obviously $K_{1}^{*}=\delta_{1} /(\mathrm{ra})$. Notice that this is the same structure as i. in previous subsection, so $p_{\beta_{1}}^{(1)}=p_{\beta}^{(2)}$ and $q_{\beta}^{(1)}=q_{\beta}^{(2)}$ thus follow.

ii. Let $\beta_{2}(z)=\log \left(\theta_{2} z+\delta_{2}\right)$. The optimal fixed deductible, $K_{2}^{*}$, must then satisfy

$$
\mathbb{E}\left[\left(\theta_{2} Z+\delta_{2}\right) \mathbb{1}_{\left\{Z \geq K_{2}\right\}}\right]=\exp \left(\operatorname{raK}_{2}\right) \mathbb{E}\left[\mathbb{1}_{\left\{Z \geq K_{2}\right\}}\right] .
$$

For the exponential distribution, we can write this criteria more explicitly as

$$
\theta_{2}\left(K_{2}+\frac{1}{\eta}\right)+\delta_{2}=\exp \left(r a K_{2}\right)
$$

which can be solved using the Lambert $W$ function,

$$
K_{2}^{*}=\frac{-1}{r a} W_{-1}\left(-\frac{r a}{\theta_{2}} \exp \left(-r a\left(\frac{\delta_{2}}{\theta_{2}}+\frac{1}{\eta}\right)\right)\right)-\frac{1}{\eta}-\frac{\delta_{2}}{\theta_{2}} .
$$

Here we use that $-r a \exp \left(-r a\left(\delta_{2} / \theta_{2}+1 / \eta\right)\right) / \theta_{2} \in[-\exp (-1), 0)$ according similar arguments as in Appendix D. (21) can for an exponential distribution be calculated as

$$
\begin{gathered}
p_{\beta_{2}}^{(1)}=\mathbb{E}\left[\exp \left(\beta_{2}(Z)\right)\left(Z-K_{2}^{*}\right)^{+}\right]=\mathbb{E}\left[\left(\theta_{2} Z+\delta_{2}\right)\left(Z-K_{2}^{*}\right)^{+}\right] \\
=\left(\frac{\theta_{2} K_{2}^{*}+\delta_{2}}{\eta}+\frac{2 \theta_{2}}{\eta^{2}}\right) \exp \left(-\eta K_{2}^{*}\right) .
\end{gathered}
$$


iii. Let $\beta_{3}(z)=\theta_{3} z+\delta_{3}$. Then the optimal fixed amount deductible level, $K_{3}^{*}$, satisfies

$$
\mathbb{E}\left[\exp \left(\theta_{3} Z+\delta_{3}\right) \mathbb{1}_{\left\{Z \geq K_{3}\right\}}\right]=\exp \left(\mathrm{raK}_{3}\right) \mathbb{E}\left[\mathbb{1}_{\left\{Z \geq K_{3}\right\}}\right] .
$$

For exponential loss distribution, the optimal deductible can be solved explicitly as

$$
K_{3}^{*}=\frac{1}{r a-\theta_{3}}\left(\log \left(\frac{\eta}{\eta-\theta_{3}}\right)+\delta_{3}\right),
$$

which exists and is positive for $r a>\theta_{3}$ and $\eta>\theta_{3}$. Furthermore, (21) can be expressed as

$$
\begin{gathered}
p_{\beta_{3}}^{(1)}=\mathbb{E}\left[\exp \left(\beta_{3}(Z)\right)\left(Z-K_{3}^{*}\right)^{+}\right]=\mathbb{E}\left[\exp \left(\theta_{3} Z+\delta_{3}\right)\left(Z-K_{3}^{*}\right)^{+}\right] \\
=\frac{\eta}{\left(\eta-\theta_{3}\right)^{2}} \exp \left(\delta_{3}\right) \exp \left(-\left(\eta-\theta_{3}\right) K_{3}^{*}\right) .
\end{gathered}
$$

\section{Numerical Illustration}

The results of the previous section are now collected and the analysis concluded by a numerical illustration. To do so values of the parameters must be chosen. We here consider an individual with a utility parameter $a=15$, subjective discount factor $\rho=10 \%$, a claim frequency $\lambda=0.01$, and losses are assumed to be exponentially distributed with parameter $\eta=0.1$. The net premium for full insurance of the nonlife risk of this individual is thus $\xi=\lambda / \eta=0.1$. Suppose also that the risk-free interest rate is $r=5 \%$.

\subsection{The impact of the pricing measure function}

We start off by visualising the impact of the pricing measure function. Firstly, we plot the loss density under the pricing measure determined by (17), and secondly, we illustrate the optimal insurance strategy of the individual. Let $f$ denote the density of the exponential distribution with parameter $\eta$, and $f^{\mathbb{Q}}$ the density under the pricing measure $\mathbb{Q}$.

When the insurance company sets its premium according to a constant pricing measure function, $\beta_{1}(z)=\delta_{1}$, it does not charge for claim size risk, and therefore the density of the claims remains unchanged, i.e. $f(z)=f^{\mathbb{Q}}(z)$. The a priori density and the pricing density is presented in Figure 1a. Instead, the claim frequency used for pricing increases to $\lambda^{\mathbb{Q}}=\lambda \exp \left(\delta_{1}\right)$. For $\delta_{1}=3.75$ the optimal fixed deductible level is then $K=5$, which is $50 \%$ of the average loss of $1 / \eta=10$. Note that for a constant pricing measure function, a fixed amount deductible is optimal for the individual, and the welfare loss in (13) is therefore zero. The optimal deductible strategy is depicted in Figure 1b.

For a log-linear pricing measure function, $\beta_{2}(z)=\log \left(\theta_{2} z+\delta_{2}\right)$, similar plots are displayed in Figure $2 \mathrm{a}$ and $2 \mathrm{~b}$. The pricing parameters are calibrated to satisfy that the individual would optimally choose a fixed amount deductible level of $K_{2}^{*}=5$ if restricted to do so. Choosing $\delta_{2}=2.5$, then this calibration leads to $\theta_{2}=2.6681$. The loss density under the pricing measure, namely $f^{\mathbb{Q}}(z)=\eta \exp (-\eta z)\left(\theta_{2} z+\delta_{2}\right) /\left(\theta_{2} / \eta+\delta_{2}\right)$, has quite a different nature than the a 


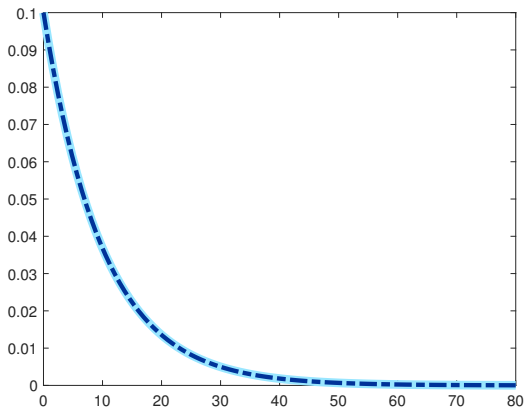

(a) The densities $f(z)$ (in solid) and $f^{\mathbb{Q}}(z)$ (in dash-dotted).

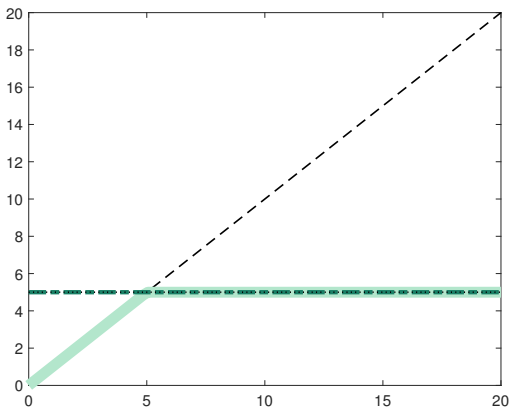

(b) The functions $g_{1}(z)$ (in dash-dotted) and $\min \left\{z, g_{1}(z)\right\}$ (in solid).

Figure 1: For a constant pricing measure function

priori exponential distribution, though still staying within the exponential family of distributions. We refer to Figure 2a. Recall from (17) that claim frequency risk is penalized by $\lambda^{\mathbb{Q}}=\lambda\left(\theta_{2} / \eta+\delta_{2}\right)$. In Figure $2 \mathrm{~b}$ the optimal flexible deductible strat-

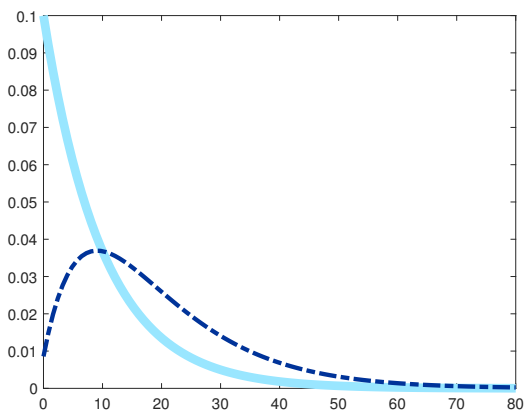

(a) The densities $f(z)$ (in solid) and $f^{\mathbb{Q}}(z)$ (in dash-dotted).

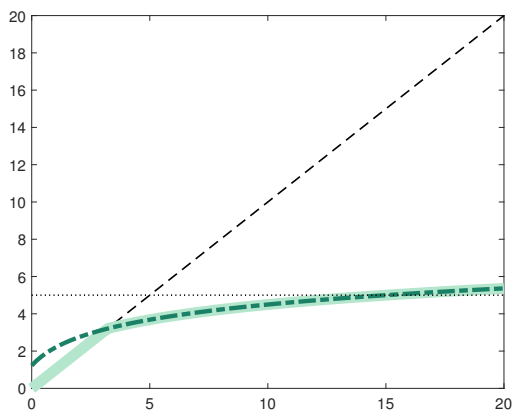

(b) The functions $g_{2}^{*}(z)$ (in dash-dotted) and $\min \left\{z, g_{2}^{*}(z)\right\}$ (in solid).

Figure 2: For a log-linear pricing measure function

egy, namely $g_{2}^{*}(z)=\log \left(\theta_{2} z+\delta_{2}\right) /(r a)$, as a function of the loss is illustrated. The optimal fixed amount deductible level for these pricing parameters also appears in the graph. We observe that the flexible insurance product yields a deductible level below the standard product for small claim sizes, whereas it slowly grows above for larger claim sizes. In this specific case, the welfare loss of the individuals being restricted to the standard product with a fixed amount deductible rather than the flexible product is $L=1.2116$, when the pricing measure function is log-linear. As mentioned previously, for this individual the net premium for full insurance is $\xi=0.1$, so relative to this, the welfare loss is $L / \xi=12.116$.

Corresponding plots for a linear pricing measure function, $\beta_{3}(z)=\theta_{3} z+\delta_{3}$, can be seen in Figure 3a and 3b. Again, the parameters $\theta_{3}=0.25$ and $\delta_{3}=1.2472$ are chosen to such that the optimal fixed deductible level is $K_{3}^{*}=5$. The density under the pricing measure is still exponential, $f^{\mathbb{Q}}(z)=\left(\theta_{3}-\eta\right) \exp \left(-\left(\eta-\theta_{3}\right) z\right)$, 
but tilted to have a heavier tail, this is apparent in Figure 3a. Claim frequency is penalised by $\lambda^{\mathbb{Q}}=\lambda \eta \exp \left(\delta_{3}\right) /\left(\eta-\theta_{3}\right)$.

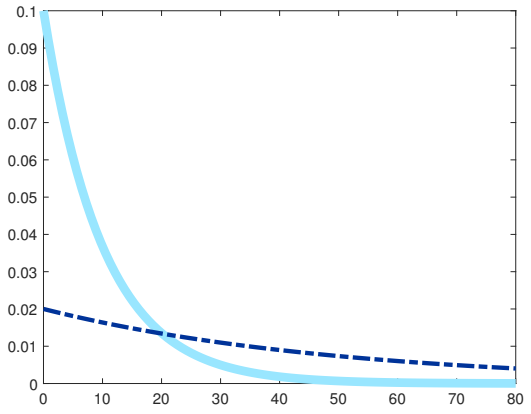

(a) The densities $f(z)$ (in solid) and $f^{\mathbb{Q}}(z)$ (in dash-dotted).

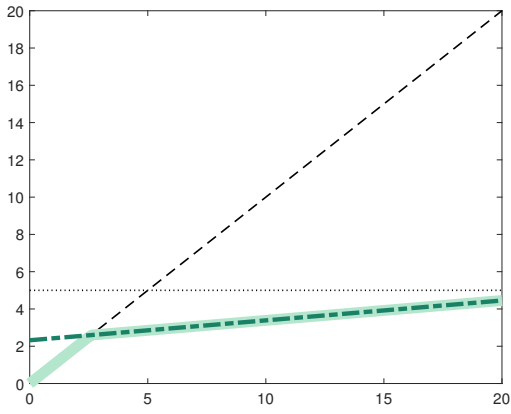

(b) The functions $g_{3}^{*}(z)$ (in dash-dotted) and $\min \left\{z, g_{3}^{*}(z)\right\}$ (in solid).

Figure 3: For a linear pricing measure function

In Figure 3b the optimal design of the deductible, $g_{3}^{*}(z)=\left(\theta_{3} z+\delta_{3}\right) /(\mathrm{ra})$, is illustrated if the individual could choose freely. Compared to being limited to a fixed amount deductible strategy, the individual would optimally choose a linearly growing strategy, which exceeds $K_{3}^{*}$ at the point $\left(r a K_{3}^{*}-\delta_{3}\right) / \theta_{3}$ (not visible on the graph). The individual's welfare loss of being restricted to the simple product rather than the flexible, when the pricing measure function is linear, is $L=16.6683$ for this choice of parameters. Relative to the cost of full insurance, the loss is then $L / \xi=166.683$ times larger.

\subsection{The welfare loss}

The welfare loss is obviously dependent on the values of the parameters. To illustrate the sensitivity towards changes in the values of these parameters as clear as possible, graphs of the relative loss $L / \xi$ are displayed in Figure 4 for a log-linear pricing measure function, and in Figure 5 for a linear pricing measure function. Each figure has four subfigures where one of the parameters vary, while the remaining are kept fixed. Figure $4 \mathrm{a}$ and $5 \mathrm{a}$ show the relative loss as a function of $\theta$, $4 \mathrm{~b}$ and $5 \mathrm{~b}$ as a function of $\delta, 4 \mathrm{c}$ and $5 \mathrm{c}$ as a function of the absolute risk aversion $a$ and, finally, $4 \mathrm{~d}$ and $5 \mathrm{~d}$ as a function of the loss parameter $\eta$. In the latter, we remark that also the net premium for full insurance varies in $\eta$. Note that the optimal fixed deductible changes as well, when varying these parameters.

For the $\log$-linear pricing measure function, $\beta_{2}(z)=\log \left(\theta_{2} z+\delta_{2}\right)$, we observe that the relative loss increases when $\theta_{2}$ increases, see Figure $4 \mathrm{a}$. This makes good sense intuitively since $\partial g_{2}^{*}(z) / \partial z=1 /\left(r a\left(z+\delta_{2} / \theta_{2}\right)\right)$, hence a larger value of $\theta_{2}$ yields a steeper slope of the optimal flexible deductible strategy, which then will deviate more from the fixed amount deductible strategy. Notice that $\delta_{2}$ has an inverse impact on $\partial g_{2}^{*}(z) / \partial z$, and we can therefore conclude the converse for $\delta_{2}$. The parameter $\delta_{2}$ also controls the intersection with the vertical axis and by raising it, a larger part of the function $g_{2}^{*}(z)$ with a steep slope will be above the identity line. Hence, as it appears in Figure $4 \mathrm{~b}$, the relative loss decreases in $\delta_{2}$. Next, 
recall that $a$ is the parameter of the exponential utility, and is thus a measure of the absolute risk aversion. An individual with risk neutral preferences ( $a$ close to zero) would not pay for insurance as she does not care about the risk. So for this type of individual it does not matter which product is supplied as long as 'no insurance' is a possibility. On the other hand, an individual with a very high degree of risk aversion ( $a$ large) would prefer to insure fully, and once again, the product structure becomes subordinate as long as a 'full insurance' (i.e. a zero deductible) can be chosen. Hence, the difference in product design is the most important for individuals with non-extreme preference, as it appears from the non-monotonicity of Figure 4c. At last, in Figure 4d the relative welfare loss is decreasing in the loss parameter $\eta$ as expected. When $\eta$ increases the tail of the loss distribution gets lighter, and claims will on average get smaller. Hence, the difference between the optimal flexible deductible strategy and the fixed amount deductible for large claim sizes affects the welfare loss less.

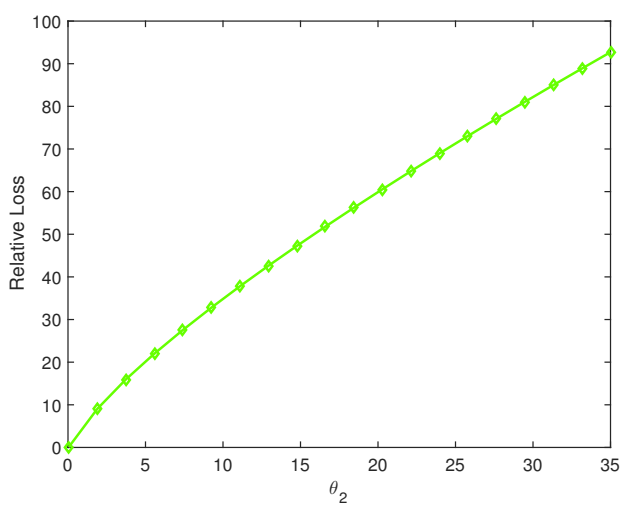

(a) Loss as function of $\theta_{2}$.

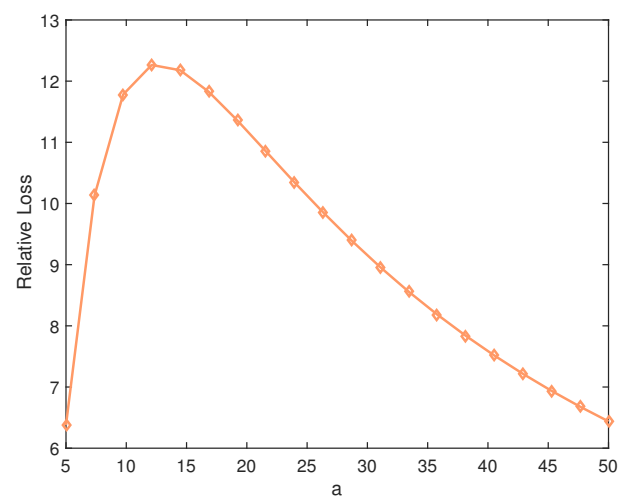

(c) Loss as function of $a$.

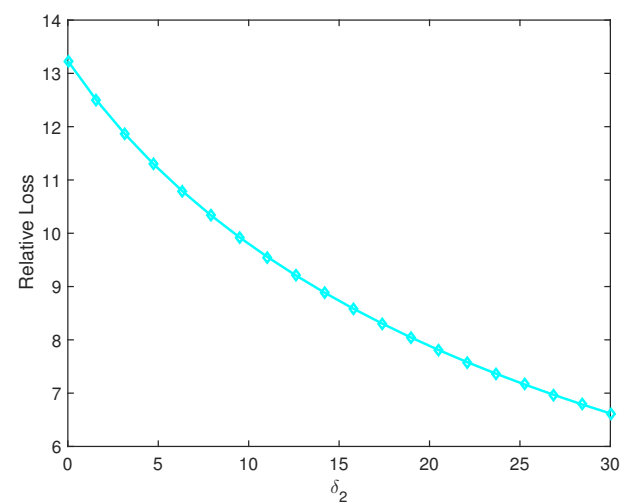

(b) Loss as function of $\delta_{2}$.

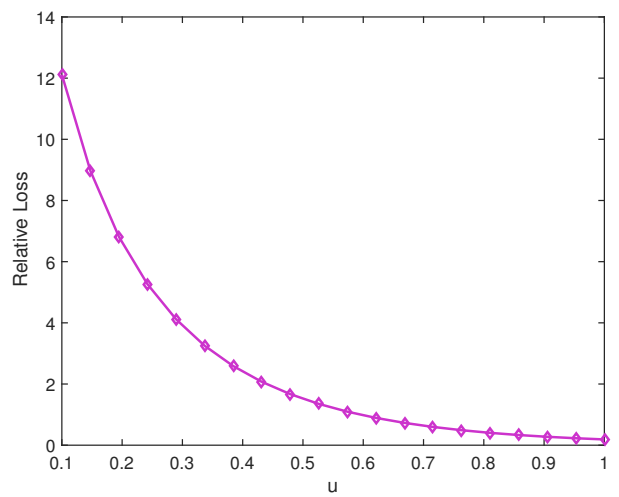

(d) Loss as function of $u$.

Figure 4: Loss function for $\beta_{2}$

The arguments for the case with a linear pricing measure function, $\beta_{3}(z)=$ $\theta_{3} z+\delta_{3}$, are similar. Since $\partial g_{3}^{*}(z) / \partial z=\theta_{3} /(\mathrm{ra})$, the parameter $\theta_{3}$ controls the slope of the optimal flexible deductible strategy. A higher value of $\theta_{3}$ yields a higher slope, so the distance to the fixed deductible will then be larger and the welfare 
loss bigger, the graph in Figure 5a is therefore increasing. If $\delta_{3}$ increases then the flexible deductible strategy will exceed the fixed deductible for lower values of the losses, and the individual is therefore forced to buy more insurance for large values of the claim if being restricted to a fixed deductible, leading to a larger welfare loss, which explains the decreasing shape of Figure 5b. For the risk aversion coefficient, the effect from being able to choose a slope on the coverage function dominates. If the individual is tending towards risk neutrality ( $a$ small), then the product design allows her to choose a high slope giving her a smaller (if not zero) insurance coverage. In contrast, for the risk averse individual ( $a$ large) that seeks a high insurance coverage, the best she can obtain in terms of slope is the fixed amount deductible (that is, zero slope), in which case the difference between the flexible product and the product with a fixed amount deductible diminishes, which explains the monotonicity in Figure 5c. The sensitivity to the loss parameter $\eta$ is similar to the second case of pricing measure function, except that here the deviation from the flexible deductible structure to the fixed amount deductible is larger for big losses, which means that the relative loss is more sensitive towards the heaviness of the loss, as we see in Figure 5d.

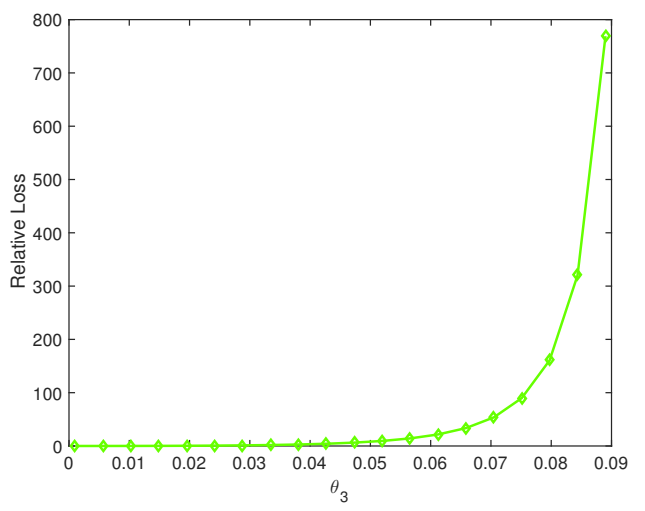

(a) Loss as function of $\theta_{3}$.

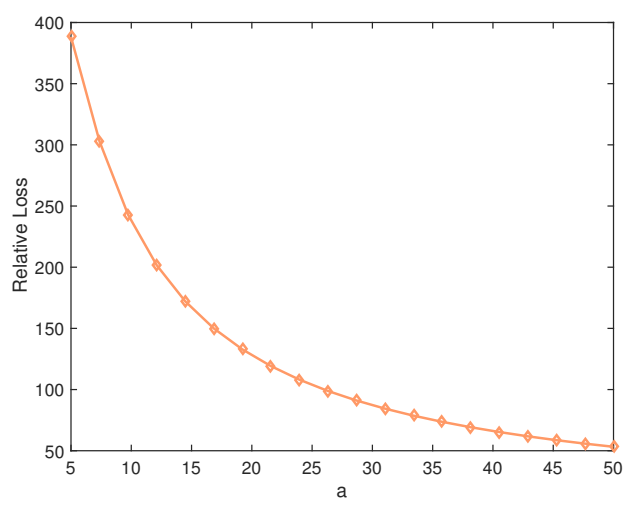

(c) Loss as function of $a$.

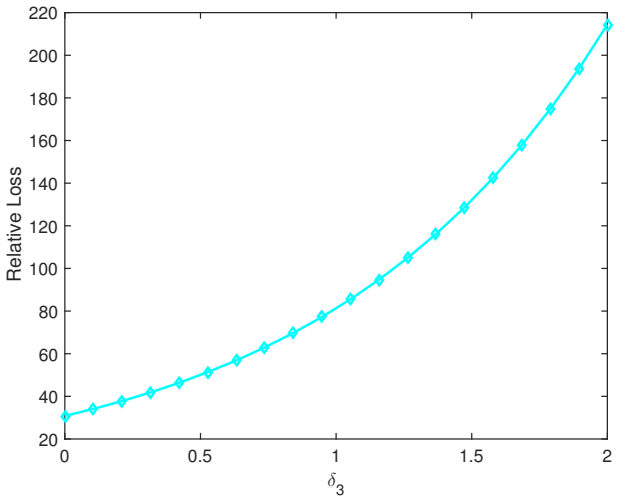

(b) Loss as function of $\delta_{3}$.

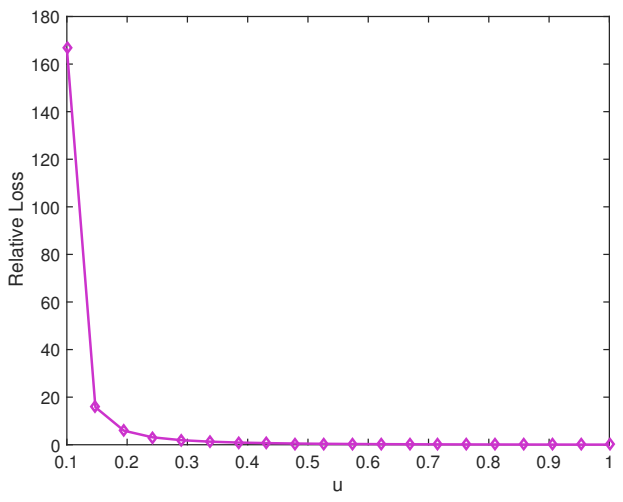

(d) Loss as function of $u$.

Figure 5: Loss function for $\beta_{3}$ 


\section{$7 \quad$ A digression on power utility}

So far we have concentrated almost exclusively on the exponential utility function. Under exponential preferences of the policyholder we could calculate explicitly both the optimal flexible deductible and the best deductible within the suboptimal class of constant deductibles. We benefited from explicit solutions, even to the suboptimal insurance position, along with the wealth-independent insurance decisions in order to obtain an explicit and wealth independent quantification of the welfare loss from being offered a suboptimal product design.

In this section we briefly touch upon the case of power utility. Power utility is a more standard formalization of individual preferences within the area of personal financial decision making. However, in order to find a solution to the optimization problem (20), it is necessary to assume that a deductible can exceed the loss, which corresponds to removing the truncation and minimum in (20). If the deductible exceeds the loss, it is interpreted as if the individual is actually betting against having a loss of that size. So if the individual finds insurance to be too expensive, then the she will not only choose not to insure, but will actually try to turn it to her advantage that the pricing is too high.

Unfortunately, we therefore do not find explicit solutions to the problem of choosing a fixed deductible, since this standard contract involves the truncation and the minimum. This prevents explicit quantification of the welfare loss from suboptimal contracts in spite of the fact that we can actually find an optimal flexible contract. Although we cannot present the best choice among the standard marketed contract, we choose here to present briefly the optimal flexible one. This serves at the same time as yet another illustration of the HJB machinery exploited in the previous sections as well as a motivating starting point for further studies in the direction.

The result (1) is developed under wealth-homogeneous assumptions on the insurance control as the compound Poisson structure is essential and it is therefore necessary to have i.i.d claim sizes $\left(Z_{i}-g_{v}\left(Z_{i}\right)\right)_{i \in \mathbb{N}}$. This works successfully for the exponential utility due to its desirable analytical properties. For a power-utility, the optimal deductible strategy depends on wealth which prevents a compound Poisson structure, but we deliberately use the result of (1), conditional on the preclaim wealth, without further notice. This should be taken as a premium principle (where the jump intensity and the claim size risk are punished separately) rather than a legitimate measure change. The premium at time $t$ is then a function of the time $t$ wealth. Another approach would be to discretize the claim sizes, introduce a pricing rate for every level of the claim sizes, and let the individual choose a distinct deductible for each of these levels. The issue with the measure change is then avoided and similar results are produced, but we will not comment further on this.

Proposition 7.1. Assume that the utility function is of the form

$$
u(c)=\frac{1}{1-\gamma} c^{1-\gamma} .
$$

for $\gamma>0, \gamma \neq 1$. Then the value function (4) can be written by

$$
V(x)=\alpha(x-\kappa)^{1-\gamma} .
$$


The optimal consumption $c^{*}$ is affine in wealth

$$
c^{*}(x)=(\alpha(1-\gamma))^{-1 / \gamma}(x-\kappa),
$$

and the optimal insurance control is given by

$$
g^{*}(x, z)=\left(1-\exp (\beta(z))^{-1 / \gamma}\right)(x-\kappa) .
$$

The parameters $\alpha$ and $\kappa$ of the value function are determined by

$$
\begin{aligned}
& \alpha=\frac{1}{(1-\gamma) \gamma^{-\gamma}}\left(\rho-r(1-\gamma)-\lambda \mathbb{E}\left[1-\exp (\beta(Z))^{-1 / \gamma}\right](1-\gamma)\right. \\
& \left.-\lambda\left(\mathbb{E}\left[\exp (\beta(Z))^{(\gamma-1) / \gamma}\right]-1\right)\right)^{-\gamma} \\
& \kappa=\frac{\lambda}{r} \mathbb{E}[\exp (\beta(Z)) Z] .
\end{aligned}
$$

Proof. We use the same approach as seen previously. We make the conjecture that the value function is of the type $V(x)=\alpha(x-\kappa)^{1-\gamma}$. First order conditions leads to the optimal choices $c^{*}$ and $g^{*}(z)$, and if we insert these back into the HJB equation and solve, we obtain $\alpha$ and $\kappa$.

Note that unlike the exponential utility case this insurance control depends on wealth. When the initial wealth approaches the present value of the cost of the claims, i.e. when $x \rightarrow \kappa$, the individual stops consuming, $c^{*}(x) \rightarrow 0$, and insures fully, $g^{*}(x, z) \rightarrow 0$ for any fixed $z$. The reason why it is so important to protect the value of full coverage is that an individual with power preference must avoid negative consumption and wealth almost surely.

Although we cannot quantify the welfare loss from a suboptimal coverage, we know that there is one, and the result of Proposition 7.1 represents an idea for product development in the non-life business. The optimal deductible is affine in the wealth. For $x \gg \kappa$, the optimal deductible is essentially linear in wealth. This could be incorporated in the insurance product or, at least, in product advice given to policy holders.

The proportionality of $g^{*}$ in $x$, namely $1-\exp (\beta(z))^{-1 / \gamma}$, has a simple structure which can also be the starting point for further product development. For the linear pricing principle, this is a constant. We note that for the linear pricing rule the optimal deductible is not a constant but a constant fraction of wealth (minus the typically relative small value of full coverage). For our variance principle the fraction becomes a linear-power function of the loss and for our Esscher principle the fraction becomes an exponential-power function. These are quite simple structures that can easily be incorporated in indemnity tables.

The limiting cases for the risk aversion are obvious. As $\gamma \rightarrow \infty, g^{*} \rightarrow 0$ for all claims and all sizes of wealth. If the individual is extremely risk averse, she avoids risk at any price and demands full protection. As $\gamma \rightarrow 0, g \rightarrow x-\kappa$ for all claim sizes. If the individual is not really risk averse, she can keep the risk at any price but only up to the point where a claim threatens her wealth in order to avoid, above all, negative wealth and consumption. 


\section{References}

Aase, K. K. (2017). "Optimal Insurance Policies in the Presence of Costs". Risks 5.3 .

Arrow, K. J. (1971). "The theory of risk aversion". Essays in the Theory of Risk Bearing, pp. 90-109.

Avanzi, B., Tu, V., and Wong, B. (2016). "A note on realistic dividends in actuarial surplus models". Risks 4.4, p. 37.

Cummins, J. D. and Mahul, O. (2004). "The demand for insurance with an upper limit on coverage". The Journal of Risk and Insurance 71.2, pp. 253-264.

Delbaen, F. and Haezendonck, J. (1989). "A martingale approach to premium calculation principles in an arbitrage free market". Insurance: Mathematics and Economics 8.4, pp. 269-277.

Embrechts, P. (2000). "Actuarial versus financial pricing of insurance". The Journal of Risk Finance 1.4, pp. 17-26.

Golubin, A. (2016). "Optimal insurance and reinsurance policies chosen jointly in the individual risk model". Scandinavian Actuarial Journal 2016.3, pp. 181197.

Grandits, P., Hubalek, F., Schachermayer, W., and Žigo, M. (2007). "Optimal expected exponential utility of dividend payments in a Brownian risk model". Scandinavian Actuarial Journal 2007.2, pp. 73-107.

Hubalek, F. and Schachermayer, W. (2004). "Optimizing expected utility of dividend payments for a Brownian risk process and a peculiar nonlinear ODE". Insurance: Mathematics and Economics 34.2, pp. 193-225.

Moore, K. S. and Young, V. R. (2006). "Optimal insurance in a continuous-time model". Insurance: Mathematics and Economics 39.1, pp. 47-68.

Perera, R. S. (2010). "Optimal consumption, investment and insurance with insurable risk for an investor in a Lévy market". Insurance: Mathematics and Economics 46.3, pp. 479-484.

Schmidli, H. (2002). "On minimizing the ruin probability by investment and reinsurance". The Annals of Applied Probability 12.3, pp. 890-907.

- (2008). Stochastic control in insurance. London: Springer-Verlag.

Thonhauser, S. and Albrecher, H. (2011). "Optimal dividend strategies for a compound Poisson process under transaction costs and power utility". Stochastic Models 27.1, pp. 120-140.

Yang, H. and Zhang, L. (2005). "Optimal investment for insurer with jump-diffusion risk process". Insurance: Mathematics and Economics 37.3, pp. 615-634.

Zhang, X. and Siu, T. K. (2009). "Optimal investment and reinsurance of an insurer with model uncertainty". Insurance: Mathematics and Economics 45.1, pp. 8188.

Zhou, C., Wu, W., and Wu, C. (2010). "Optimal insurance in the presence of insurer's loss limit". Insurance: Mathematics and Economics 46.2, pp. 300307.

Zou, B. and Cadenillas, A. (2014). "Explicit solutions of optimal consumption, investment and insurance problems with regime switching". Insurance: Mathematics and Economics 58, pp. 159-167. 


\section{A The impact of pricing on the individual's decision}

In this section we solve the optimization of the policy holder for a particularly simple pricing rule. It serves as a motivating example for the more abstract calculations in Section 4. We are going to show very clearly and explicitly with this example that for a specific pricing rule, the size of the claim is indeed, in general, relevant for calculating the optimal deductible. It is natural to speak of the pricing rule studied in this section as being based on a change of measure which is piecewise constant in the size of the loss. Then the optimal deductible indeed also becomes piecewise constant in general.

Let $0=\ell_{1}<\ell_{2}<\ldots<\ell_{n-1}<\ell_{n}=\infty$ be given, such that $\left[\ell_{1}, \ell_{2}\right), \ldots,\left[\ell_{n-1}, \ell_{n}\right)$ is a finite partition of $\mathbb{R}^{+}$. When a loss of size $z$ occurs, it falls into one of the regions $\left[\ell_{i}, \ell_{i+1}\right)$ for $i=1, \ldots, n-1$. Assume that the individual can choose a distinct deductible for each region, i.e. a deductible $K_{i}$ when $z$ takes value in $\left[\ell_{i}, \ell_{i+1}\right)$. In this case the insurance product is characterized by

$$
g_{\left(K_{1}, \ldots, K_{n}\right)}(z)=\sum_{i=1}^{n-1} K_{i} \mathbb{1}_{\left\{z \in\left[\ell_{i}, \ell_{i+1}\right)\right\}} .
$$

The theory of space-decomposition allows us to split, correspondingly, the compound Poisson process $\left(A_{t}\right)_{t \geq 0}$ describing the losses of the individual (without insurance) into $n$ compound Poisson processes with jump rate $\lambda_{i}=\lambda \mathbb{P}\left(Z \in\left[\ell_{i}, \ell_{i+1}\right)\right)$ and jump sizes in $\left[\ell_{i}, \ell_{i+1}\right)$ for $i=1, \ldots, n-1$, respectively. Assume that the insurance company prices each of these risks individually with pricing rates $\lambda_{1}^{Q}, \ldots, \lambda_{n}^{Q}$, where typically $\lambda_{i+1}^{Q} / \lambda_{i+1}>\lambda_{i}^{Q} / \lambda_{i}$ (large claims constitute larger risks and should be charged accordingly). We can now solve the optimization problem of the policyholder for a given partition of the pricing rule.

Proposition A.1. Assume that the insurance company applies a piecewise constant pricing rule as described above. Then the value function is of type $V(x)=$ $-\exp (-\operatorname{rax}) / \alpha$ and the optimal controls are

$$
c^{*}=r x-\frac{1}{a} \log \left(\frac{r a}{\alpha}\right) \text { and } K_{i}^{*}=\frac{1}{r a} \log \left(\frac{\lambda_{i}^{Q}}{\lambda_{i}}\right) \text { for } i=1, \ldots, n .
$$

Proof. The HJB equation is in this case

$$
\begin{aligned}
\sup \{- & \rho V(x)+\left(r x-c-\sum_{i=1}^{n-1} \lambda_{i}^{\mathbb{Q}} \mathbb{E}\left[\left(Z-K_{i}\right)^{+} \mid Z \in\left[\ell_{i}, \ell_{i+1}\right)\right]\right) V_{x}(x) \\
& \left.+\sum_{i=1}^{n-1} \lambda_{i} \mathbb{E}\left[V\left(x-\min \left\{Z, K_{i}\right\}\right)-V(x) \mid Z \in\left[\ell_{i}, \ell_{i+1}\right)\right]+u(c)\right\}=0 .
\end{aligned}
$$

Since the nature of the problem is similar to the previous section, we again guess that the value function is of type $V(x)=-\exp (-\operatorname{rax}) / \alpha$. The first order condition with respect to consumption then leads to the same structure as seen before,

$$
c^{*}=r x-\frac{1}{a} \log \left(\frac{r a}{\alpha}\right),
$$


The Leibniz integral rule is used to find the first order condition with respect to the $i$ 'th deductible level,

$$
\lambda_{i}^{\mathbb{Q}} \mathbb{E}\left[\mathbb{1}_{\left\{Z_{i}>K \mid Z_{i} \in\left[\ell_{i}, \ell_{i+1}\right)\right\}}\right]-\lambda_{i} \mathbb{E}\left[\mathbb{1}_{\left\{Z_{i}>K \mid Z_{i} \in\left[\ell_{i}, \ell_{i+1}\right)\right\}}\right] \exp \left(r a K_{i}\right)=0 .
$$

Rearranging and isolating yields

$$
K_{i}^{*}=\frac{1}{r a} \log \left(\frac{\lambda_{i}^{\mathbb{Q}}}{\lambda_{i}}\right) \text { for } i=1, \ldots, n .
$$

Inserting the optimal controls in the HJB we obtain the supremum, and we can then solve for

$$
\begin{aligned}
\alpha=\frac{1}{r a} \exp & \left(\frac{1}{r}\left(\rho-\sum_{i=1}^{n} \lambda_{i}\left(\mathbb{E}\left[\exp \left(r a \min \left\{Z, K_{i}^{*}\right\}\right) \mid Z \in\left[\ell_{i}, \ell_{i+1}\right)\right]-1\right)\right)\right. \\
& \left.-a \sum_{i=1}^{n} \lambda_{i}^{\mathbb{Q}} \mathbb{E}\left[\left(Z-K_{i}^{*}\right)^{+} \mid Z \in\left[\ell_{i}, \ell_{i+1}\right)\right]-1\right) .
\end{aligned}
$$

Proposition A.1 shows in a simple way how the price of the insurance coverage affects the optimal extent of coverage. The more expensive the insurance is, measured by the pricing ratio $\lambda_{i}^{\mathbb{Q}} / \lambda_{i}$, the larger a deductible is optimal for the individual. For the exponential utility case the part is as simple as the logarithm of the pricing ratio times a constant which contains the level of risk aversion.

A special case arises if there is only one 'piece' and piecewise constant really means constant. This corresponds to the expected value pricing principle since the value of a contract is proportional to its expectation with a constant of proportionality equal to $\lambda^{\mathbb{Q}} / \lambda$. As previously argued, if the pricing is based on the expected value premium principle, then a fixed amount deductible is optimal. Proposition A.1 repeats this result for the special case of exponential utility and determines the constant deductible level to be

$$
K^{*}=\frac{1}{r a} \log \left(\frac{\lambda^{\mathbb{Q}}}{\lambda}\right) .
$$

The $n$ piecewise constant deductible case is a special case of the measure transformation with pricing measure function

$$
\beta(z)=\sum_{i=1}^{n} \delta_{i} \mathbb{1}_{z \in\left[\ell_{i}, \ell_{i+1}\right)} \text { where } \delta_{i}=\log \left(\frac{\lambda_{i}^{Q}}{\lambda_{i}}\right) \text { for all } i .
$$

\section{B Proof of Proposition 4.1}

Proof. To abbreviate the notation, let $f_{\beta}(t)=\exp (-\lambda t \mathbb{E}[\exp (\beta(Z))-1])$ and $h_{v}(z)=\left(z-g_{v}(z)\right)^{+}$. Now simply consider the characteristic function 


$$
\begin{aligned}
\mathbb{E}^{\mathbb{Q}(\beta)} & {\left[\exp \left(\mathrm{i} s \cdot \sum_{i=1}^{N_{t}} h_{v}\left(Z_{i}\right)\right)\right] } \\
& =\mathbb{E}\left[\exp \left(\sum_{i=1}^{N_{t}} \beta\left(Z_{i}\right)-\lambda t \mathbb{E}[\exp (\beta(Z))-1]\right) \exp \left(\mathrm{i} s \cdot \sum_{i=1}^{N_{t}} h\left(Z_{i}\right)\right)\right] \\
& =f_{\beta}(t) \mathbb{E}\left[\prod_{i=1}^{N_{t}} \frac{\exp \left(\beta\left(Z_{i}\right)\right) \exp \left(\mathrm{i} s \cdot h_{v}\left(Z_{i}\right)\right)}{\mathbb{E}[\exp (\beta(Z))]} \mathbb{E}[\exp (\beta(Z))]\right] \\
& =f_{\beta}(t) \mathbb{E}\left[\mathbb{E}\left[\prod_{i=1}^{N_{t}} \frac{\exp \left(\beta\left(Z_{i}\right)\right) \exp \left(\mathrm{i} s \cdot h_{v}\left(Z_{i}\right)\right)}{\mathbb{E}[\exp (\beta(Z))]} \mathbb{E}[\exp (\beta(Z))] N_{t}\right]\right] \\
& =f_{\beta}(t) \mathbb{E}\left[\mathbb{E}[\exp (\beta(Z))]^{N_{t}} \mathbb{E}\left[\frac{\exp (\beta(Z)) \exp \left(\mathrm{i} s \cdot h_{v}(Z)\right)}{\mathbb{E}[\exp (\beta(Z))]}\right]^{N_{t}}\right] \\
& =f_{\beta}(t) \exp \left(\lambda t\left(\mathbb{E}[\exp (\beta(Z))] \mathbb{E}\left[\frac{\exp (\beta(Z))}{\mathbb{E}[\exp (\beta(Z))]} \exp \left(\mathrm{i} s \cdot h_{v}\left(Z_{i}\right)\right)\right]-1\right)\right) \\
& =\exp \left(\lambda t \mathbb{E}[\exp (\beta(Z))]\left(\mathbb{E}\left[\frac{\exp (\beta(Z))}{\mathbb{E}[\exp (\beta(Z))]} \exp \left(\mathrm{i} s \cdot h_{v}\left(Z_{i}\right)\right)\right]-1\right)\right) .
\end{aligned}
$$

This is the characteristic function of a compound Poisson process with characteristics (17). From this, the expected value (18) follows directly.

C Calculations for $\beta_{1}(z)=\delta_{1}$

$$
\begin{aligned}
\mathbb{E} & {\left[\exp \left(r a \cdot \min \left\{Z, \delta_{1} /(r a)\right\}\right)\right] } \\
& =\mathbb{P}\left(A_{1}\right) \mathbb{E}\left[\exp (r a Z) \mid A_{1}\right]+\mathbb{P}\left(A_{1}^{c}\right) \mathbb{E}\left[\exp \left(\delta_{1}\right) \mid A_{1}^{c}\right] \\
& =\mathbb{E}\left[\exp (r a Z) \mathbb{1}_{\left\{Z<\delta_{1} /(r a)\right\}}\right]+\mathbb{E}\left[\exp \left(\delta_{1}\right) \mathbb{1}_{\left\{Z \geq \delta_{1} /(r a)\right\}}\right] \\
& =\int_{0}^{\delta_{1} /(r a)} \eta \exp ((r a-\eta) z) d z+\exp \left(\frac{r a-\eta}{r a} \delta_{1}\right) \\
& =\frac{r a}{r a-\eta} \exp \left(\frac{r a-\eta}{r a} \delta_{1}\right)-\frac{\eta}{r a-\eta} . \\
\mathbb{E} & {\left[\exp (\beta(Z))(Z-g(Z))^{+}\right]=\mathbb{P}\left(A_{1}^{c}\right) \exp \left(\delta_{1}\right) \mathbb{E}\left[\left(Z-\delta_{1} /(r a)\right) \mid A_{1}^{c}\right] } \\
& =\mathbb{E}\left[\exp \left(\delta_{1}\right)\left(Z-\delta_{1} /(r a)\right) \mathbb{1}_{\left\{Z \geq \delta_{1} /(r a)\right\}}\right] \\
& =\exp \left(\delta_{1}\right) \int_{\delta /(r a)}^{\infty} \eta \exp (-\eta z)\left(z-\delta_{1} /(r a)\right) \mathrm{d} z \\
& =\frac{1}{\eta} \exp \left(\frac{r a-\eta}{r a} \delta_{1}\right) .
\end{aligned}
$$




\section{Calculations $\beta_{2}(z)=\log \left(\theta_{2} z+\delta_{2}\right)$}

Let $\tilde{Z}=\theta_{2} Z+\delta_{2}$ and $\hat{Z}=-r a \tilde{Z} / \theta_{2}$. The event $A_{2}=\left\{Z<\log \left(\theta_{2} Z+\delta_{2}\right) /(r a)\right\}$ can then be rewritten as

$$
\begin{aligned}
A_{2}=\{Z & \left.<\log \left(\theta_{2} Z+\delta_{2}\right) /(r a)\right\}=\left\{\exp (r a Z)<\theta_{2} Z+\delta_{2}\right\} \\
= & \left\{\exp \left(r a \tilde{Z} / \theta_{2}\right) \exp \left(-\delta_{2} r a / \theta_{2}\right)<\tilde{Z}\right\} \\
= & \left\{\hat{Z} \exp (\hat{Z})<-r a \exp \left(-\delta_{2} r a / \theta_{2}\right) / \theta_{2}\right\} \\
= & \left\{\hat{Z}>W_{-1}\left(-r a \exp \left(-\delta_{2} r a / \theta_{2}\right) / \theta_{2}\right)\right\},
\end{aligned}
$$

where we use that $-r a \exp \left(-\delta_{2} r a / \theta_{2}\right) / \theta_{2} \in[-\exp (-1), 0)$, which is necessary in order for $W_{-1}$ to be defined. The upper boundary is trivial, whereas the lower is a bit less so. First, we recognize that $-x \exp \left(-\delta_{2} x\right)>-x \exp (-x)$ for any values of $x$ and $\delta_{2} \geq 1$. Next, we see that $-x \exp (-x)$ obtains its minimum for $x=1$, hence $-x \exp (-x)>-\exp (-1)$.

Translated back to $Z$ using substitution, we can now conclude that

$$
A_{2}=\{Z<Q\} \text { where } Q=-\frac{1}{r a} W_{-1}\left(-\frac{r a}{\theta_{2}} \exp \left(-\delta_{2} \frac{r a}{\theta_{2}}\right)\right)-\frac{\delta_{2}}{\theta_{2}}
$$

We therefore get

$$
\begin{aligned}
\mathbb{E}\left[\exp \left(r a \cdot \min \left\{Z, \log \left(\theta_{2} Z+\delta_{2}\right) /(r a)\right\}\right)\right] & \\
= & \mathbb{P}\left(A_{2}\right) \mathbb{E}\left[\exp (r a Z) \mid A_{2}\right]+\mathbb{P}\left(A_{2}^{c}\right) \mathbb{E}\left[\theta_{2} Z+\delta_{2} \mid A_{2}^{c}\right] \\
= & \int_{A_{2}} \eta \exp ((r a-\eta) z) d z+\int_{A_{2}^{c}} \eta\left(\theta_{2} z+\delta_{2}\right) \exp (-\eta z) d z \\
= & \int_{0}^{Q} \eta \exp ((r a-\eta) z) d z+\int_{Q}^{\infty} \eta\left(\theta_{2} z+\delta_{2}\right) \exp (-\eta z) d z \\
= & \frac{\eta}{r a-\eta}(\exp ((r a-\eta) Q)-1)+\left(\theta_{2} Q+\delta_{2}+\frac{\theta_{2}}{\eta}\right) \exp (-\eta Q) . \\
\mathbb{E}[(\theta Z+ & \left.\left.\delta_{2}\right)\left(Z-\log \left(\theta_{2} Z+\delta_{2}\right) /(r a)\right)^{+}\right] \\
= & \mathbb{P}\left(A_{2}^{c}\right) \mathbb{E}\left[\left(\theta_{2} Z+\delta_{2}\right)\left(Z-\log \left(\theta_{2} Z+\delta_{2}\right) /(r a)\right) \mid A_{2}^{c}\right] \\
= & \int_{A_{2}^{c}} \eta\left(\theta_{2} z+\delta_{2}\right)\left(z-\log \left(\theta_{2} z+\delta_{2}\right) /(r a)\right) \exp (-\eta z) d z \\
= & \int_{Q}^{\infty} \eta\left(\theta_{2} z+\delta_{2}\right)\left(z-\log \left(\theta_{2} z+\delta_{2}\right) /(r a)\right) \exp (-\eta z) d z \\
= & \exp (-\eta Q)\left(\theta_{2} Q^{2}+\left(\delta_{2}+2 \frac{\theta_{2}}{\eta}\right) Q-\frac{1}{\eta}\left(\frac{\theta_{2}}{r a}-\delta_{2}\right)+2 \frac{\theta_{2}}{\eta^{2}}\right. \\
& \quad-\frac{1}{r a} \frac{\theta_{2}}{\eta} \exp \left(\frac{\eta}{\theta_{2}}\left(\theta_{2} Q+\delta_{2}\right)\right) E_{1}\left(\frac{\eta}{\theta_{2}}\left(\theta_{2} Q+\delta_{2}\right)\right) \\
& \left.-\frac{1}{r a}\left(\theta_{2} Q+\delta_{2}+\frac{\theta_{2}}{\eta}\right) \log \left(\theta_{2} Q+\delta_{2}\right)\right) .
\end{aligned}
$$




\section{E Calculations for $\beta_{3}(z)=\theta_{3} z+\delta_{3}$}

$\mathbb{E}\left[\exp \left(r a \cdot \min \left\{Z,\left(\theta_{3} Z+\delta_{3}\right) /(r a)\right\}\right)\right]$

$$
\begin{aligned}
& =\int_{A_{3}} \eta \exp (r a z) \exp (-\eta z) \mathrm{d} z+\int_{A_{3}^{c}} \eta \exp \left(\theta_{3} z+\delta_{3}\right) \exp (-\eta z) \mathrm{d} z \\
= & \int_{0}^{\delta_{3} /\left(r a-\theta_{3}\right)} \eta \exp (r a z) \exp (-\eta z) \mathrm{d} z+\int_{\delta_{3} /\left(r a-\theta_{3}\right)}^{\infty} \eta \exp \left(\theta_{3} z+\delta_{3}\right) \exp (-\eta z) \mathrm{d} z \\
= & \frac{\eta}{\eta-r a}\left(1-\exp \left(-(\eta-r a) \frac{\delta_{3}}{r a-\theta_{3}}\right)\right)+\frac{\eta}{\eta-\theta_{3}} \exp \left(-(\eta-r a) \frac{\delta_{3}}{r a-\theta_{3}}\right) .
\end{aligned}
$$

$\mathbb{E}\left[\exp \left(\theta_{3} Z+\delta_{3}\right)\left(Z-\left(\theta_{3} Z+\delta_{3}\right) /(r a)\right)^{+}\right]$

$$
\begin{aligned}
& =\int_{A_{3}^{c}} \exp \left(\theta_{3} z+\delta_{3}\right)\left(z-\left(\theta_{3} z+\delta_{3}\right) /(r a)\right) \mathrm{d} z \\
& =\int_{\delta_{3} /\left(r a-\theta_{3}\right)}^{\infty} \exp \left(\theta_{3} z+\delta_{3}\right)\left(z-\left(\theta_{3} z+\delta_{3}\right) /(r a)\right) \mathrm{d} z \\
& =\exp \left(-(\eta-r a) \frac{\delta_{3}}{r a-\theta_{3}}\right) \frac{\left(r a-\theta_{3}\right) \eta}{r a\left(\eta-\theta_{3}\right)^{2}} .
\end{aligned}
$$

